\title{
The Downwind Hemisphere of the Heliosphere: Eight Years of IBEX-Lo Observations
}

\author{
A. Galli ${ }^{1}$ (1), P. Wurz $^{1}$ (1), N. A. Schwadron ${ }^{2}$, H. Kucharek $^{2}$, E. Möbius $^{2}$, M. Bzowski $^{3}$ (1), \\ J. M. Sokół ${ }^{3}$, M. A. Kubiak ${ }^{3}$, S. A. Fuselier ${ }^{4}$, H. O. Funsten ${ }^{5}$ (D) and D. J. McComas ${ }^{6}$ (D) \\ ${ }^{\mathrm{i}}$ Physics Institute, University of Bern, Bern, 3012, Switzerland \\ ${ }^{2}$ University of New Hampshire, Durham, NH 03824, USA \\ ${ }^{3}$ Space Research Centre, Polish Academy of Sciences, Warsaw, 00-716, Poland \\ ${ }^{4}$ Southwest Research Institute and University of Texas, San Antonio, TX 78228, USA \\ ${ }^{5}$ Los Alamos National Laboratory, Intelligence and Space Research Division, Los Alamos, NM 87545, USA \\ ${ }^{6}$ Princeton University, Department of Astrophysical Sciences and Office of the Vice President for the Princeton Plasma \\ Physics Laboratory, Princeton, NJ 08540, USA \\ Received 2017 October 2; revised 2017 November 3; accepted 2017 November 3; published 2017 December 5
}

\begin{abstract}
We present a comprehensive study of energetic neutral atoms (ENAs) of $10 \mathrm{eV}$ to $2.5 \mathrm{keV}$ from the downwind hemisphere of the heliosphere. These ENAs are believed to originate mostly from pickup protons and solarwind protons in the inner heliosheath. This study includes all low-energy observations made with the Interstellar Boundary Explorer over the first eight years. Because the protons around $0.1 \mathrm{keV}$ dominate the plasma pressure within the inner heliosheath in downwind direction, these ENA observations offer the unique opportunity to constrain the plasma properties and dimensions of the heliosheath where no in situ observations are available. We first derive energy spectra of ENA intensities averaged over time for 49 macropixels covering the entire downwind hemisphere. The results confirm previous studies regarding integral intensities and the roll-over around $0.1 \mathrm{keV}$ energy. With the expanded data set, we now find that ENA intensities at 0.2 and $0.1 \mathrm{keV}$ seem to anti-correlate with solar activity. We then derive the product of total plasma pressure and emission thickness of protons in the heliosheath to estimate lower limits on the thickness of the inner heliosheath. The temporally averaged ENA intensities support a rather spherical shape of the termination shock and a heliosheath thickness between 150 and 210 au for most regions of the downwind hemisphere. Around the nominal downwind direction of $76^{\circ}$ ecliptic longitude, the heliosheath is at least 280 au thick. There, the neutral hydrogen density seems to be depleted compared to upwind directions by roughly a factor of 2 .
\end{abstract}

Key words: ISM: general - plasmas - solar wind - Sun: heliosphere

\section{Introduction}

The Interstellar Boundary Explorer (IBEX) has been continually observing the interaction of the heliosphere with the surrounding interstellar medium since 2009 January (McComas et al. 2009). The scientific payload consists of two energetic neutral atom (ENA) imagers: IBEX-Lo (Fuselier et al. 2009) and IBEX-Hi (Funsten et al. 2009). This study expands previous studies of energy spectra of ENAs observed at low energies (Fuselier et al. 2014; Galli et al. 2014, 2016) in terms of time (2009-2016) and spatial coverage (the entire downwind hemisphere is included in the analysis and interpretation). The ENAs analyzed here are believed to originate predominantly from the shocked solar wind and pickup protons beyond the termination shock. The ENAs thus allow us to sample the source plasma populations over a vast region of the heliosphere not accessible to in situ measurements.

The ultimate goal of this study is to better understand the heliosphere, its boundaries, and the properties of plasma populations in the heliosheath. McComas et al. (2013), Zirnstein et al. (2016a) investigated the Port Tail and Starboard Tail lobes in IBEX-Hi maps of ENA intensities: at solar-wind energies or higher, regions of depleted ENA emissions appear in two lobes $30-100^{\circ}$ apart from the nominal downwind direction. Will a similar structure appear at lower ENA energies? Observations of neutral hydrogen column densities via Ly $\alpha$ absorption (Wood et al. 2014) indicate that the heliotail cannot be deflected by more than $20^{\circ}$ with respect to the nominal downwind direction $\left(\lambda_{\mathrm{ecl}}=76^{\circ}, \beta_{\mathrm{ecl}}=-5^{\circ}\right)$ defined by the interstellar flow (McComas et al. 2015). We will demonstrate in this paper that the low-energy ENAs around $0.2 \mathrm{keV}$ sampled with $I B E X$-Lo must be taken into account to understand the complex geometry of the heliosheath in the downwind direction. The parent ions of this energy range dominate the plasma pressure. With the energy spectra of ENAs measured over the full energy range of $I B E X$, we will be able to derive observational constraints on the geometry, cooling lengths, and neutral density of the heliosheath, albeit at a very coarse spatial resolution.

We first present the data set and how we corrected ENA spectra with the corresponding uncertainties from IBEX-Lo observations (Sections 2 and 3). We then summarize (in Section 4) the results of the spectra, discuss the implications of our results on the downwind hemisphere of the heliosphere (Section 5), and conclude the paper with a summary and outlook (Section 6).

\section{Data Set}

IBEX-Lo is a single-pixel ENA camera (Fuselier et al. 2009). Neutral atoms enter the instrument through a collimator, which defines the nearly conical field-of-view of 6.5 full width at half maximum. A fraction of the incident ENAs then scatter off a charge-conversion surface as negative ions. These ions pass through an electrostatic energy analyzer and are accelerated into a time-of-flight mass spectrometer that features a triple coincidence detection scheme. Apart from negatively ionized 
ENAs, IBEX-Lo can also detect $\mathrm{H}^{-}$and $\mathrm{O}^{-}$sputtered off the conversion surface by interstellar neutrals (ISN) and ENAs of solar wind energy or higher (Park et al. 2016). IBEX-Lo measures ENAs at eight different energy passbands with central energies at $0.015,0.029,0.055,0.11,0.209,0.439$, 0.872 , and $1.821 \mathrm{keV}$ (Fuselier et al. 2009).

The observation times for this study include the first eight years of IBEX-Lo triple coincidence data of hydrogen ENAs, corresponding to 16 seasonal maps from 2009 January until 2016 October. Ram maps (in which $I B E X$, in its orbit around Earth, moves toward the emission source) of the downwind hemisphere are created from measurements acquired over April-October each year, while anti-ram downwind maps are created from measurements during October-April. The ram and anti-ram distinction is important to this study because the proper motion of the spacecraft is not negligible compared to the ENA velocity in the inertial frame. Ram observations therefore have a significantly better signal-to-background ratio than anti-ram observations, as discussed in Section 3. The data set includes only the times with the lowest background levels; measurements affected by high-electron background were excluded with the method described earlier by Galli et al. (2016).

Two basic limitations of the data set must be kept in mind. First, IBEX spent the four months from July until mid-October every year inside the bow shock of Earth's magnetosphere. As a consequence, no observations with sufficiently low background level were obtained. This causes a data gap over ecliptic longitudes from $0^{\circ}$ to $120^{\circ}$, covering most of the downwind hemisphere in the ram direction. Ram observations of slow ENAs have a better signal-to-background ratio than anti-ram observations, which results in smaller error bars (see Section 3). Second, observations after 2012 July exhibited a lower signal-to-noise ratio: the post-acceleration of $I B E X$-Lo had to be reduced in 2012 July and the background caused by the terrestrial magnetosphere and the solar wind was elevated from 2012-2016 during the maximum of solar activity (Galli et al. 2015).

We assumed that the energy of detected hydrogen ENAs at 1 au heliocentric distance is the same as their energy at their place of origin in the heliosheath. This is substantiated during solar minimum (2009-2011) because their energy loss due to solar radiation pressure, sustained while travelling from the plasma source to $I B E X$, is nearly compensated by the energy gain due to solar gravity-even for low energies at tens of $\mathrm{eV}$ (Bzowski 2008). During solar maximum conditions (2012-2015), a $22 \mathrm{eV}$ hydrogen ENA emitted far away from the Sun reaches $1 \mathrm{au}$ at an apparent energy of $15 \mathrm{eV}$ (center of the lowest-energy bin of $I B E X-\mathrm{Lo}$ ), and a $36 \mathrm{eV}$ ENA is decelerated to $29 \mathrm{eV}$ (corresponding to the center of energy bin 2) (Bzowski 2008). For higher energies, the differences are even smaller. We neglected these energy shifts in the study because we detected no consistent increase of ENA intensity at 15 and $29 \mathrm{eV}$ when we compared ENA maps from the first four years with the maps from 2013-2016. We also verified for 2009 (solar minimum conditions) and 2014 (solar maximum conditions) that no significant bias in corrected ENA intensities occurred at a spatial resolution of $24^{\circ} \times 24^{\circ}$ if we replaced our default correction and mapping algorithm with a different algorithm based on simulation runs with the Warsaw test particle model (Sokółet al. 2015a). In the latter approach, we
Table 1

Ubiquitous Background Hydrogen Count Rates

\begin{tabular}{lcc}
\hline \hline Energy Bin & Center Energy & Background Count Rate in s $^{-1}$ \\
\hline 1 & $0.015 \mathrm{keV}$ & $0.0067 \pm 0.0015$ \\
2 & $0.029 \mathrm{keV}$ & $0.0075 \pm 0.001$ \\
3 & $0.055 \mathrm{keV}$ & $0.0076 \pm 0.0018$ \\
4 & $0.110 \mathrm{keV}$ & $0.0074 \pm 0.002$ \\
5 & $0.209 \mathrm{keV}$ & $0.0012 \pm 0.0012$ \\
6 & $0.439 \mathrm{keV}$ & $0.0002 \pm 0.0002$ \\
7 & $0.872 \mathrm{keV}$ & Not detectable \\
8 & $1.821 \mathrm{keV}$ & Not detectable \\
\hline
\end{tabular}

Note. Background count rates observed in the lower energies of IBEX-Lo for the years 2013-2016, after the post-acceleration of IBEX-Lo was reduced. The background was quantified by demanding that the heliospheric ENA signal in the solar inertial reference frame (between $300^{\circ}$ and $360^{\circ}$ ecliptic longitude) should be equal for ram and anti-ram observations after background subtraction.

corrected the ENA measurements for the proper motion of $I B E X$, solar gravity, and the solar radiation pressure.

As in Galli et al. (2016), we used data from a single season and one energy bin, and constructed the map of differential intensities of heliospheric hydrogen ENAs (in units of $\mathrm{cm}^{-2} \mathrm{sr}^{-1} \mathrm{~s}^{-1} \mathrm{keV}^{-1}$ ) at 100 au heliocentric distance in the inertial reference frame, with respect to the Sun, at a spatial resolution of $6^{\circ} \times 6^{\circ}$. The ENA intensities were first corrected for average sputtering contributions to the ENA measurements and the ubiquitous background (values as stated in Galli et al. (2015) for 2009-2012; values for 2013-2016 as stated in Table 1). After this subtraction, the remaining ENA intensities were corrected for the energydependent survival probability of ENAs (see Appendices in Galli et al. 2016; McComas et al. 2017) and for the proper motion of the spacecraft relative to the Sun as described by Galli et al. (2016). We also compensated for the cylindrical distortion in our map projection for viewing directions at ecliptic latitudes beyond $\pm 60^{\circ}$ (see Figure 1).

Using the corrected ENA intensity maps, we then calculated the median ENA intensity inside macropixels of size $24^{\circ} \times 24^{\circ}$ pixels that are constructed from arrays of four $6^{\circ}$ pixels in latitude and four $6^{\circ}$ pixels in longitude. This mesh of macropixels is shown in Figure 1, overlaid on the uncorrected ENA maps measured at $0.029 \mathrm{keV}$ (top panel) and $0.872 \mathrm{keV}$ (bottom panel). At solar wind energy, the ENA intensity map is dominated by the ENA Ribbon (McComas et al. 2014; Schwadron et al. 2014), at low energies, it is dominated by the primary and secondary populations of ISN helium and hydrogen (Möbius et al. 2012; Saul et al. 2013; Kubiak et al. 2014; McComas et al. 2015). The macropixels cover the entire downwind hemisphere, except the polar pixels (studied by Reisenfeld et al. 2016), with the edges situated at ecliptic longitudes of $120^{\circ}, 96^{\circ}, 72^{\circ}, 48^{\circ}, 24^{\circ}, 0^{\circ}$, $336^{\circ}$, and $312^{\circ}$, and over the ecliptic latitude range $-84^{\circ}$ to $+84^{\circ}$. The construction of the macropixels was determined by balancing the coverage of as much of the downwind hemisphere as possible with equally sized regions, retaining sufficient spatial resolution to identify variability across and between large emission structures, and achieving a sufficient signal-to-noise ratio per region for statistically significant results. The last criterion meant that we wanted at least $N=10$ ENA counts per season per macropixel at each energy bin (refer to Section 3 for 

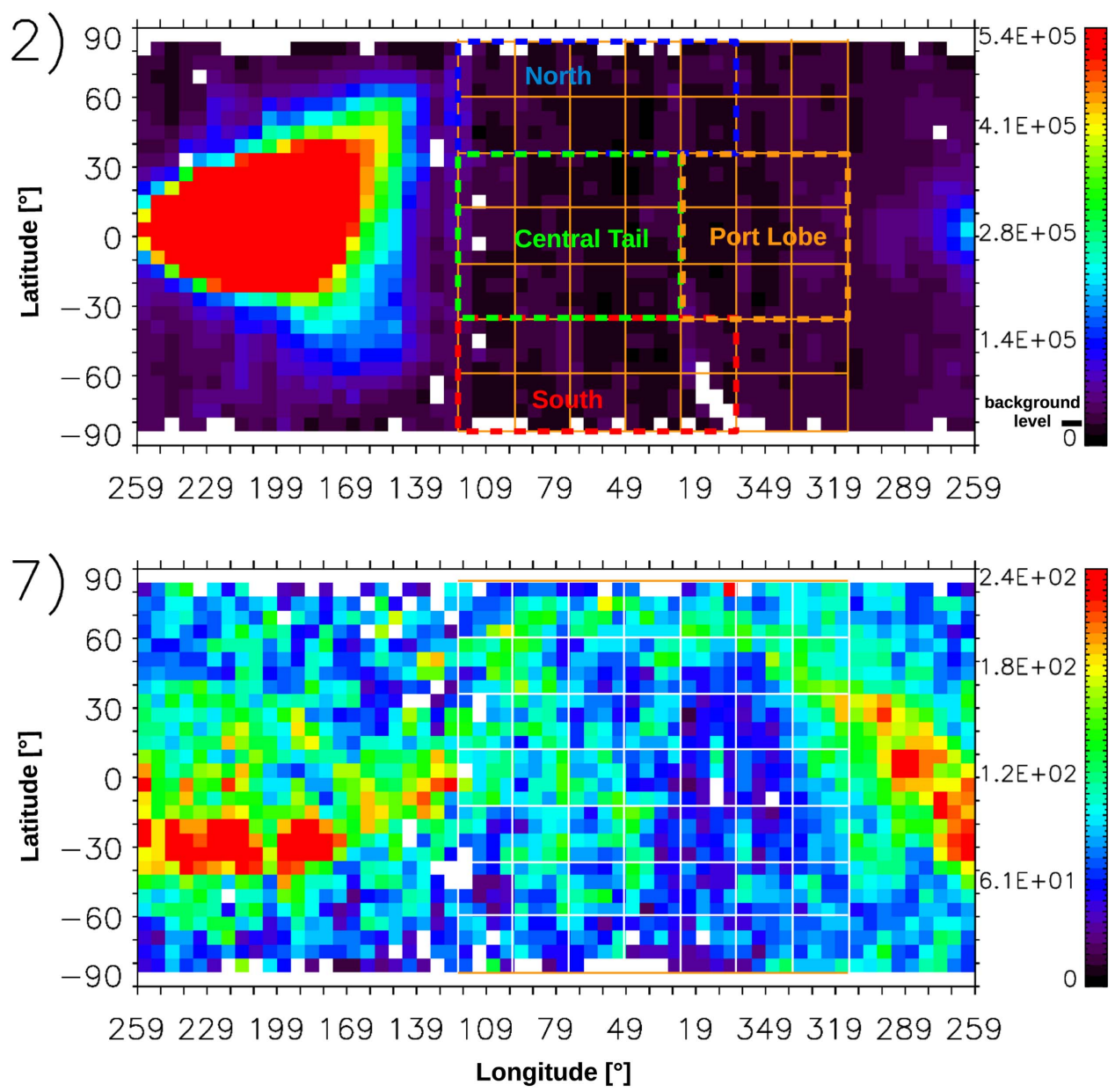

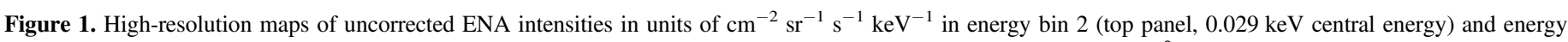

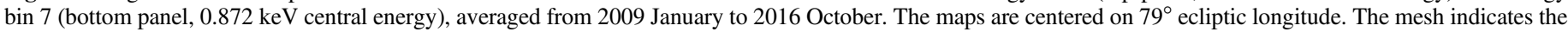

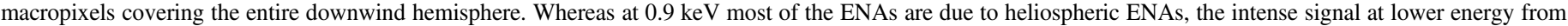

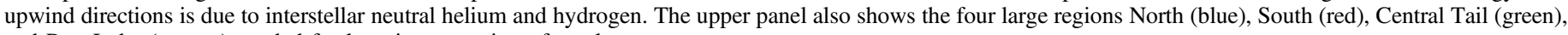
and Port Lobe (orange) needed for later interpretation of results.

an explanation). If less than four of the sixteen $6^{\circ} \times 6^{\circ}$ pixels had a valid intensity, for instance because of data-acquisition gaps, the macropixel for that season and energy was omitted from analysis. Zero intensity, on the other hand, was accepted; such cases occurred at low energies where the measured count rates did not exceed the average background count rates. These pixels are colored black in the top panel of Figure 1. Zero-count pixels provide a threshold sensitivity of the instrument; these results should not be interpreted as the absence of ENA emission. We account for this in our error analysis, which is described later.

Unless we study the evolution with time, the ENA intensity per macropixel is the median over all available seasonal median values, implying eight ram and anti-ram observations during 2009-2016 for ecliptic longitudes $0^{\circ}$ to $312^{\circ}$ and eight anti-ram observations during $2009-2016$ for $120^{\circ}$ to $0^{\circ}$. A median ENA intensity per macropixel must be based on three independent seasonal values; otherwise, that macropixel is omitted from analysis. For interpretation of the results, we grouped the macropixels into the four larger regions shown in the upper panel of Figure 1. The value attributed to such a region is the median intensity calculated over all macropixels.

Contrary to previous studies (Galli et al. 2014, 2016), we did not a priori exclude any pixels with anomalously high count rates. Such a cutoff would exclude most of the intense ISN inflow of helium and hydrogen, and would also eliminate some pointlike background sources. However, any cutoff criterion for this study would be arbitrary and the ENA results might be biased to low intensities and optimistic uncertainties because the latter were computed from the observed variability of intensity. We thus accepted all pixels and verified that the new median ENA spectrum is identical within error bars to the downwind spectrum in Galli et al. (2016) for the same observation period of 2009-2012. A separation of the globally distributed heliospheric ENA emission and the ISN atoms is currently not feasible because we do not have models of the extended populations of ISN helium and hydrogen in all IBEX-Lo energy bins. The Warm Breeze model by Kubiak et al. (2016), for example, assumes a simplistic physical scenario. A more sophisticated model of the Warm Breeze, originating in the outer heliosheath due to charge exchange collisions between interstellar $\mathrm{He}$ and $\mathrm{He}^{+}$(Bzowski et al. 2017), is only available for energy bin 2 (around $29 \mathrm{eV}$ ). Fortunately, the ISN inflow hardly affects the results, as will be shown in the results section. This non-interference is enabled by our choice of observation direction, which is restricted to hydrogen ENAs from the downwind hemisphere, i.e., looking away from the ISN inflow direction (see Figure 1).

We also investigated signal-to-noise filters as employed by Park et al. (2016) to exclude single pixels from uncorrected 
seasonal maps. However, at low energy most individual pixels have a low signal-to-noise ratio and the absolute counts per pixel are on the order of unity. As a result, a signal-to-noise ratio filter was either ineffective or resulted in many excluded macropixels in the corrected maps. Finally, we investigated the comparison of raw counts in individual macropixels across different seasons to identify and possibly exclude anomalous pixels before ENA intensity correction. However, this was found to be inappropriate because the survival probability at low energies may change by more than $30 \%$ within one year. We therefore must correct the ENA intensities for survival probability and the spacecraft motion before we can meaningfully construct and compare macropixel averages, variability, and outliers.

\section{Uncertainties and Error Bars}

The corrected ENA intensity of IBEX-Lo measurements is affected both by statistical uncertainty due to the small number of counts collected during one season and by systematic errors introduced by background sources (counts caused by signals other than heliospheric hydrogen ENAs) and calibration errors. The first group of uncertainties can be reduced if we average over a larger region and/or over more seasons of observations. We quantified the various uncertainties associated with ENA intensity per macropixel the following way:

1. The pixel-by-pixel standard deviation inside a single macropixel over one season could be due to a real smallscale spatial variability or due to low count statistics. The latter is more plausible, as the observed standard deviation agrees with the relative uncertainty expected from a Poisson distribution with $1 / \sqrt{N}$, whereby $N$ equals the number of counts per pixels. This uncertainty increases markedly for lower energy, where fewer counts are available. The ratios of the pixel-by-pixel standard deviation versus median ENA intensity (for the case of an ENA signal distinguishable against background for ram observations) calculate to $1,1.2,0.8,0.5,0.3$, and 0.3 for the energy bins at $0.55,0.11,0.209,0.439,0.872$, and $1.821 \mathrm{keV}$. The most limiting case at $0.11 \mathrm{keV}$, with an average 0.7 counts (!) per entire season per $6^{\circ} \times 6^{\circ}$ pixel, explains why we organized the measurements into macropixels containing 16 single pixels. This way, we achieved a statistical uncertainty $1 / \sqrt{N}<30 \%$ at all energies for a discernible ENA signal.

2. The single count limit must be considered for averaged measurements that include multiple zero-count pixels for which both error propagation and empirical variability no longer apply: Introducing an artificial map with background count rate +1 count per season $(\approx 0.0015$ cnts $\mathrm{s}^{-1}$ ) everywhere, we assessed the single count limit in corrected ENA intensities after corrections for survival probability and Compton-Getting effect: Table 2 lists these lower limits imposed by the single count limit. In the worst case (lowest energy, anti-ram, ecliptic plane), no ENA intensity below $10^{6} \mathrm{~cm}^{-2} \mathrm{sr}^{-1} \mathrm{~s}^{-1} \mathrm{keV}^{-1}$ can be distinguished against the background level, even after combining the counts from 16 single pixels! For higher energies and for regions outside the ecliptic plane (weaker Compton-Getting effect), the single count limit becomes irrelevant compared to other uncertainties. Generally, the single count limit at low energies for a
Table 2

Implications of Single Count Limits on Corrected ENA Intensity

\begin{tabular}{lclr}
\hline \hline $\begin{array}{l}\text { Energy } \\
\text { Bin }\end{array}$ & $\begin{array}{c}\text { Ecliptic } \\
\text { Latitude }\end{array}$ & $\begin{array}{l}\text { Relative Motion of } \\
\text { Spacecraft }\end{array}$ & $\begin{array}{r}\text { Corrected ENA Intensity } \\
\text { in } \mathrm{cm}^{-2} \mathrm{sr}^{-1} \mathrm{~s}^{-1} \mathrm{keV}^{-1}\end{array}$ \\
\hline 1 & $0^{\circ}$ & ram & 1000 \\
1 & $45^{\circ}$ & ram & 300 \\
1 & $0^{\circ}$ & anti-ram & $10^{6}$ \\
1 & $45^{\circ}$ & anti-ram & 2500 \\
2 & $0^{\circ}$ & ram & 80 \\
2 & $45^{\circ}$ & ram & 80 \\
2 & $0^{\circ}$ & anti-ram & 2000 \\
2 & $45^{\circ}$ & anti-ram & 250 \\
3 & $0^{\circ}$ & ram & 56 \\
3 & $45^{\circ}$ & ram & 45 \\
3 & $0^{\circ}$ & anti-ram & 200 \\
3 & $45^{\circ}$ & anti-ram & 110 \\
\hline
\end{tabular}

single season agrees with the pixel-to-pixel standard deviation presented in point 1. Both uncertainties originate from low count statistics.

3. To estimate the error of the ENA intensity within a macropixel over all different seasons, we relied on the empirical variability between the medians from season to season. As previously stated, a minimum of three different seasons was required to calculate a meaningful spread. Because the seasonal median values are usually not normally distributed (and with some zero values), we calculated $16 \%$ and $84 \%$ quantiles of seasonal values instead of the simple standard deviation. These quantiles represent our best estimate for the $1 \sigma$ lower and upper error bar. They approach the classic standard deviation when the distribution of seasonal values approaches a normal distribution. The different seasons constitute statistically independent measurements. Nevertheless, we did not divide the error bar estimated from the season-to-season difference by the square root of seasons because the variability may be due to systematic errors (e.g., background sources or time variations), rather than only to statistical errors. The resulting error bars of the averaged signal thus represent the full encountered variability and are rather conservative.

The medians of the spatial spreads per macropixel reflect the statistical uncertainty of a single season (see point 1). That uncertainty becomes negligible compared to the systematic errors when we average over all available seasons.

4. Finally, the absolute calibration uncertainty is $\pm 30 \%$ for a given energy bin. This is irrelevant for analysis and interpretation of maps of a single energy bin. However, quantitative comparison of intensities across multiple energies requires inclusion of this uncertainty. It will be considered the minimum uncertainty for entries in an energy spectrum.

The default error bars associated with the ENA intensity per macropixel in the following section will be the $\pm 1 \sigma$ empirical error bars across different seasons, except if we study temporal evolution over individual seasons. In that case, the variability per macropixel or per region will serve as an error estimate. Figure 2 illustrates the default error bars. For this figure, we sampled the spectrum at the macropixel centered at $\lambda_{\text {ecl }}=$ $336^{\circ} \ldots 312^{\circ}$ and $\beta_{\text {ecl }}=36^{\circ} \ldots 60^{\circ}$, which was covered both with 


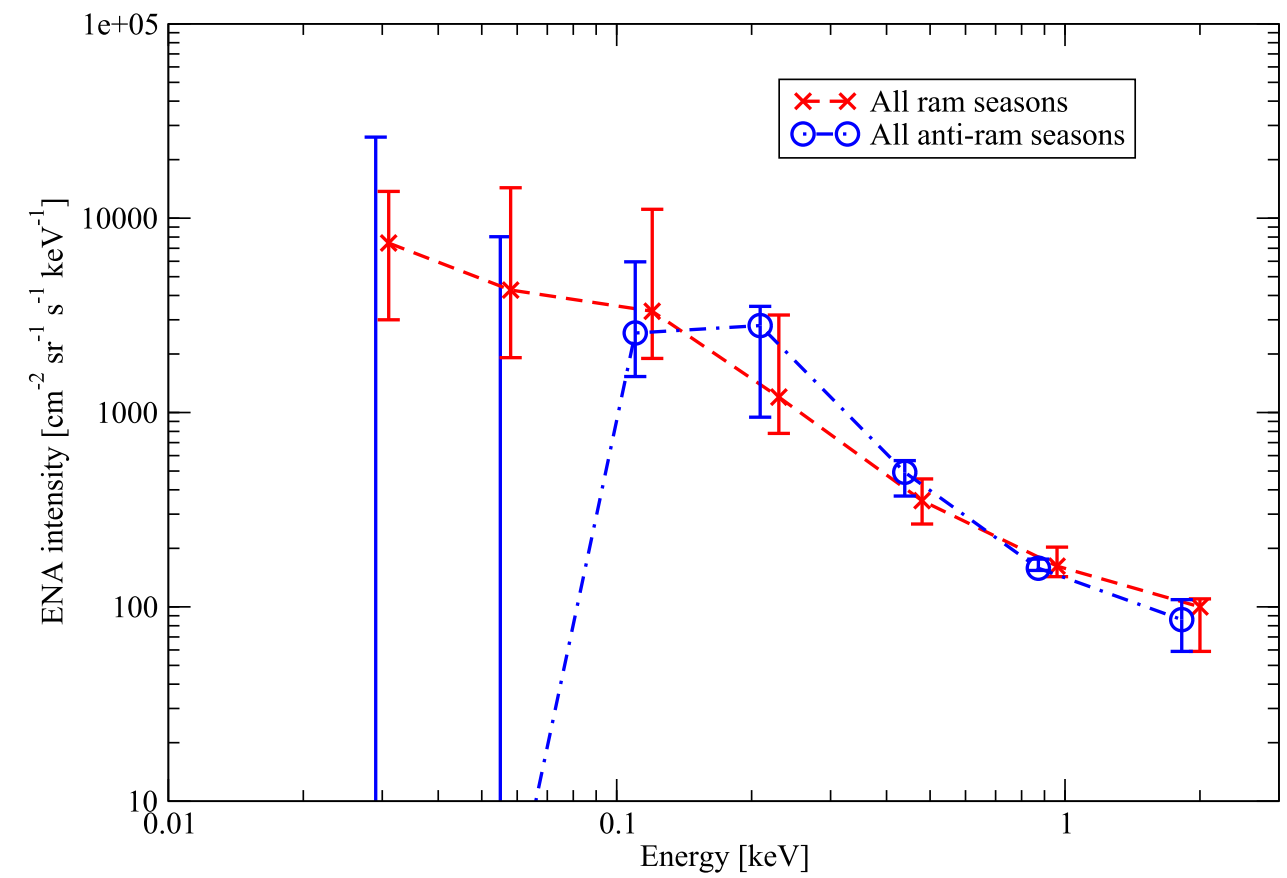

Figure 2. Energy spectra of heliospheric ENA intensity in the inertial reference frame at 100 au heliocentric distance. This figure shows the ENA energy spectrum for the identical macropixel (centered at $\lambda_{\mathrm{ecl}}=324^{\circ}, \beta_{\mathrm{ecl}}=48^{\circ}$ ), but sampled in ram (red) and anti-ram (blue) direction.

ram and anti-ram observations. This example also demonstrates the challenge of using anti-ram observations at low energies.

\section{Results}

Let us first study the spatial distribution and energy spectra of the heliospheric ENAs averaged over the entire observation time before discussing potential temporal trends and contributions by ISN at the lowest energies.

\subsection{Average ENA Intensities Over All Eight Years of Measurements}

The left columns of Figures 3 and 4 show the macropixel maps (defined by the macropixel mesh of Figure 1) of corrected ENA intensities, averaged over all 16 available seasons. The right columns show the relative uncertainty $\left(\sigma_{j} / j\right)$ of each macropixel; if the intensity is zero, the ratio of $\left(\sigma_{j} / j\right)$ is set to unity. Figure 3 features the lower energies (energy bins 1 to 4 ), while Figure 4 shows the higher energies (energy bins 5 to 8 ). Even at this coarse resolution, the ENA Ribbon shows up to the north at solar wind energies (Figure 4, energy bins 7 and 8). At intermediate energies $(0.1,0.2$, and $0.4 \mathrm{keV})$ a few pixels of high ENA intensity occur in the ecliptic, but they are interceded with other pixels of low ENA intensity. The only stable spatial feature at 0.1 to $0.9 \mathrm{keV}$ is the very low ENA intensity from the south pole region. At energies even lower (bins 1-3 from 0.015 to $0.055 \mathrm{keV}$ ), the downwind hemisphere appears uniformly dim in ENA emission, with few macropixels having an ENA intensity significantly exceeding the background level. The exceptions are situated toward the poles because of better statistics (Reisenfeld et al. 2016) and because the correction factors due to the Compton-Getting effect and the survival probability are notably smaller than at low latitudes.

From these maps, we calculated the power-law exponent or spectral index $\gamma$ of the ENA intensity spectrum above the roll-over energy for each macropixel:

$$
j(E)=j_{0}\left(E / E_{0}\right)^{-\gamma} \text {. }
$$

The resulting map of spectral indices between energy bins 7 and 8 ( 0.9 to $1.8 \mathrm{keV}$, corresponding to solar wind energy) is shown in Figure 5. The spectral index is ordered according to ecliptic latitude for these energies (in our case $\gamma=0.3+$ $\cos ^{0.8}(\beta)$ ), whereas the spectral index does not notably vary with latitude at energies below $0.9 \mathrm{keV}$. At the roll-over energy (typically $0.1 \mathrm{keV}$, see below), the sign of the spectral index changes and the ENA intensity starts decreasing with decreasing energy.

The dependence of spectral index on latitude for solar wind energy confirms IBEX-Hi observations by Desai et al. (2015), who found a similar latitudinal ordering for both upwind and downwind regions at high ENA energies. In contrast to the data presented here, Desai et al. (2015) and Zirnstein et al. (2017) observed this latitudinal ordering only above $2 \mathrm{keV}$. The latitudinal ordering of the energy spectrum probably reflects the production mechanism of the corresponding ENAs: ENAs with $1 \mathrm{keV}$ or above likely are the neutralized solar wind. Lowerenergy ENAs originate from other sources in the heliosheath that have lost the latitudinal ordering of the solar wind speed. For ENA energies below $0.1 \mathrm{keV}$, the trace-back times of the parent ions (see following subsection) are so long that ENAs measured at $I B E X$ contain a mixture of ENAs generated during solar maximum and solar minimum conditions.

At these lower energies, the spectral index of ENA intensities, averaged over all macropixels, is $1.3 \pm 0.2$ between 0.4 and $0.9 \mathrm{keV}, 2.1 \pm 0.5$ between 0.2 and $0.4 \mathrm{keV}, 1.4 \pm 0.9$ between 0.1 and $0.2 \mathrm{keV}$, and $0.2 \pm 0.7$ between 0.055 and $0.11 \mathrm{keV}$, with no notable dependence on latitude or longitude. This generalizes the statement in our previous paper (Galli et al. 2016) that the energy spectrum of ENAs from a few 

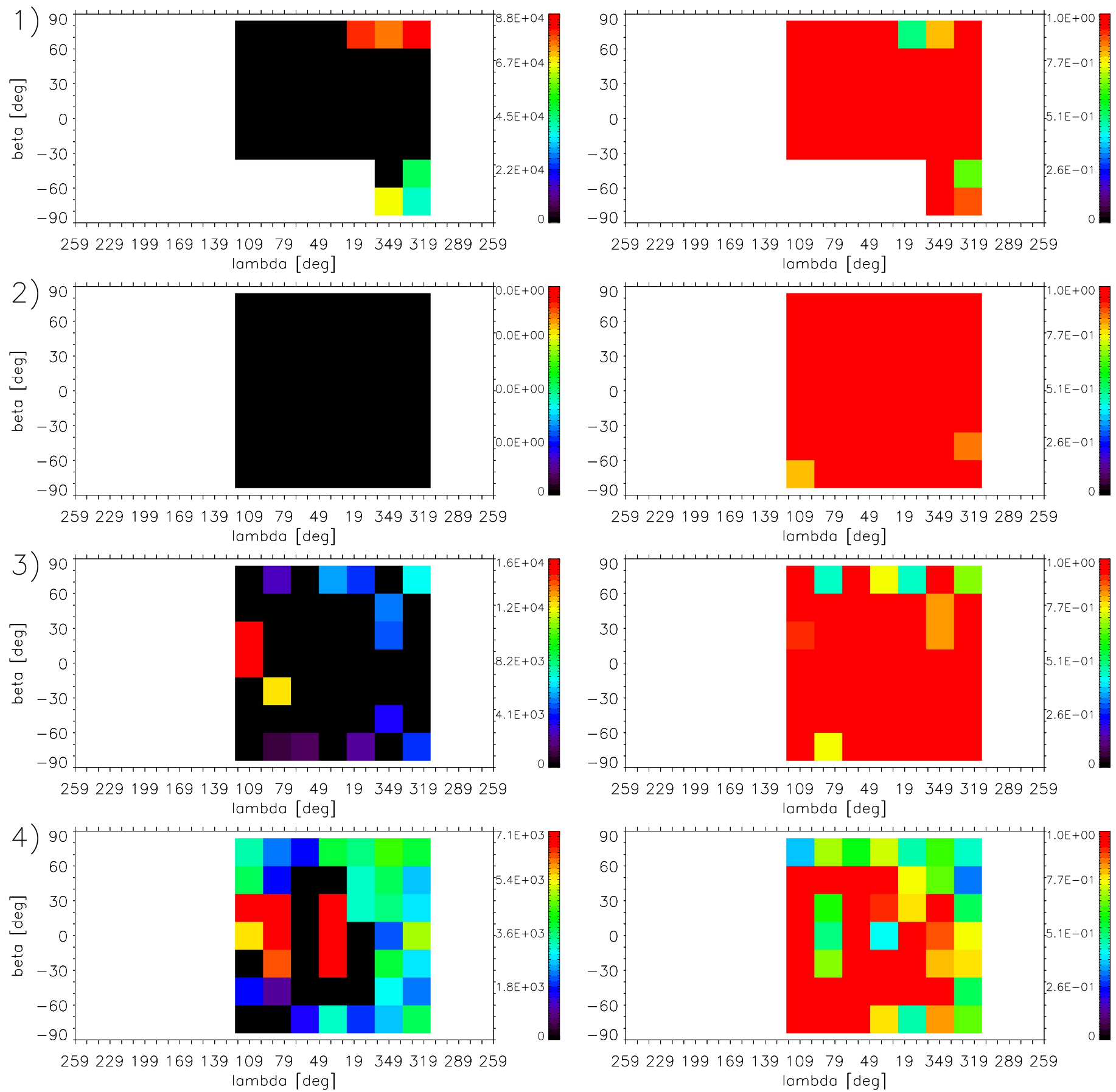

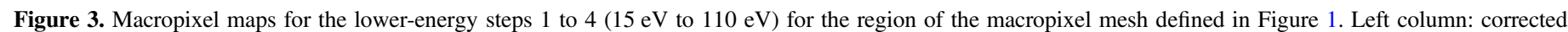

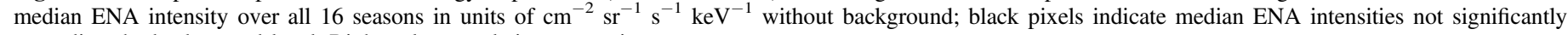
exceeding the background level. Right column: relative uncertainty.

specific downwind regions turns over around $100 \mathrm{eV}$ in the whole downwind hemisphere. Unfortunately, we cannot directly compare this roll-over to upwind directions. There, the inflow of ISN hides the much weaker signal of heliospheric ENAs below $130 \mathrm{eV}$. For the directions toward Voyager 1 and Voyager 2 (upwind hemisphere outside ecliptic plane), the observed ENA spectra appear to be higher than for the downwind hemisphere at low energies (see Figure 5 in Galli et al. (2016)). This implies additional sources of heliospheric ENAs at energies below $100 \mathrm{eV}$ from the upwind hemisphere, with $j \approx 10^{4} \mathrm{~cm}^{-2} \mathrm{sr}^{-1} \mathrm{~s}^{-1} \mathrm{keV}^{-1}$ at 29 and $15 \mathrm{eV}$ (Galli et al. 2016). However, the lower limit of these ENA intensities is zero and the emission intensity is below the instrument threshold, so we cannot rule out the possibility that a similar roll-over around $100 \mathrm{eV}$ also applies to heliospheric ENAs from the upwind hemisphere.

From here onward, let us reduce the amount of data to be interpreted. Discussing the spectral shape and temporal trends of all 49 macropixels independently is not only impractical, but also pointless: the uncertainty of the spectral index for a single macropixel (caused by the uncertainties of ENA intensity at the neighboring energies) is similar to the fitted value itself, even 

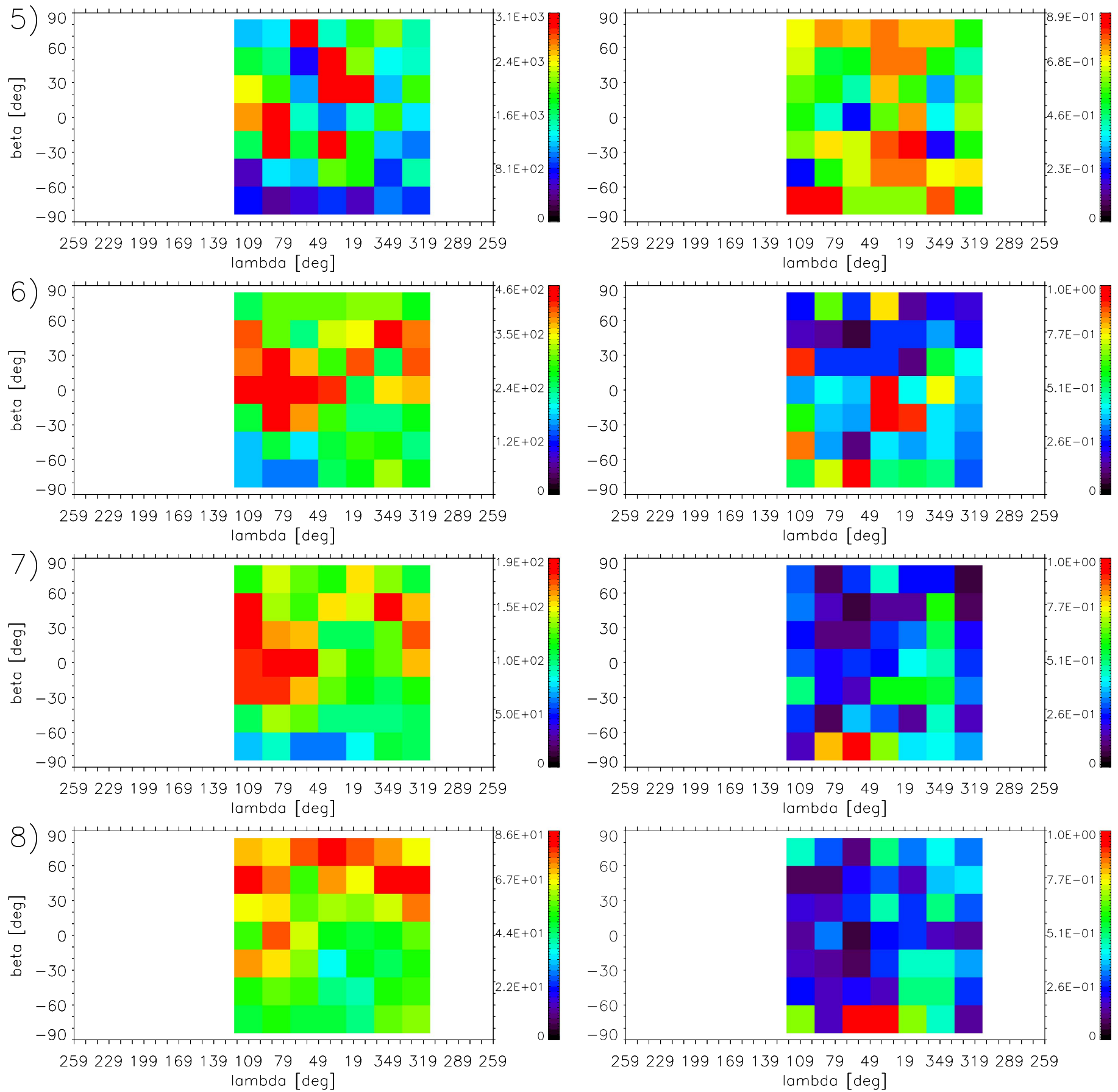

Figure 4. Macropixel maps for the higher energy bins 5 to $8(209 \mathrm{eV}$ to $1.821 \mathrm{keV})$ for the region of the macropixel mesh defined in Figure 1 ; same format as Figure 3.

for solar wind energies. Guided by Figure 5, we have thus organized the downwind macropixels into just four large regions (see upper panel of Figure 1): "North" $\left(\lambda=120^{\circ} \ldots 0^{\circ}, \beta=36^{\circ} \ldots 84^{\circ}, 10\right.$ macropixels $)$, "South" $\left(\lambda=120^{\circ} \ldots 0^{\circ}, \beta=-84^{\circ} \ldots-36^{\circ}, 10\right.$ macropixels $)$, and the two regions in the ecliptic plane: "Central Tail" $\left(\lambda=120^{\circ} \ldots 24^{\circ}, \beta=-36^{\circ} \ldots+36^{\circ}, 12\right.$ macropixels $)$ and "Port Lobe" $\left(\lambda=24^{\circ} \ldots 312^{\circ}, \beta=-36^{\circ} \ldots+36^{\circ}, 9\right.$ macropixels). We separate the Central Tail from the Port Lobe region because the latter may also contain ISN hydrogen and helium in the two lowest-energy bins. For the same reason, the four macropixels to the north and south of the Port Lobe region were excluded from the North and South regions. The nautical term "Port Tail lobe" for the area between $50^{\circ}$ and $0^{\circ}$ at the flank of the heliotail was introduced by McComas et al. (2013) to discuss the shape of the heliotail from IBEX-Hi observations. Heliospheric ENAs from the opposite region, the starboard lobe at $120^{\circ}-150^{\circ}$, could not be distinguished at low energies against the inflow of ISN (see upper panel in Figure 1).

We wanted to expand the energy range of the ENA intensity spectrum for the discussion of plasma pressure in the heliosheath (see Section 5). We therefore synthesized and added IBEX-Hi spectra from the most recent Data Release 10 (McComas et al. 2017), which represents seven-year averages 


\begin{tabular}{|r|r|r|r|r|r|r|r|}
\hline $72^{\circ}$ & 0.68 & 1.02 & 0.66 & 0.49 & 0.94 & 0.84 & 0.73 \\
\hline $48^{\circ}$ & 1.15 & 0.81 & 1.14 & 1.06 & 1.04 & 1.11 & 0.68 \\
\hline $24^{\circ}$ & 1.44 & 1.20 & 1.40 & 0.71 & 0.74 & 0.97 & 1.18 \\
\hline $0^{\circ}$ & 1.58 & 1.36 & 1.65 & 1.37 & 1.27 & 1.27 & 1.42 \\
\hline$-24^{\circ}$ & 1.22 & 1.35 & 1.36 & 1.72 & 1.12 & 1.13 & 1.08 \\
\hline$-48^{\circ}$ & 0.89 & 1.22 & 1.17 & 1.10 & 1.14 & 0.90 & 0.88 \\
\hline$-72^{\circ}$ & 0.58 & 0.71 & 0.42 & 0.40 & 0.91 & 0.86 & 0.76 \\
\hline$\beta / / \lambda$ & $108^{\circ}$ & $84^{\circ}$ & $60^{\circ}$ & $36^{\circ}$ & $12^{\circ}$ & $348^{\circ}$ & $324^{\circ}$ \\
\hline
\end{tabular}

Figure 5. Spectral indices $\gamma$ independently fitted between 0.9 and $1.8 \mathrm{keV}$ for each of the 49 macropixels of this study. Flat energy spectra with small $\gamma$-values are colored red and orange, steep energy spectra are colored bluish. The ENA energy spectrum is steeper in the ecliptic plane and flattens toward the poles.

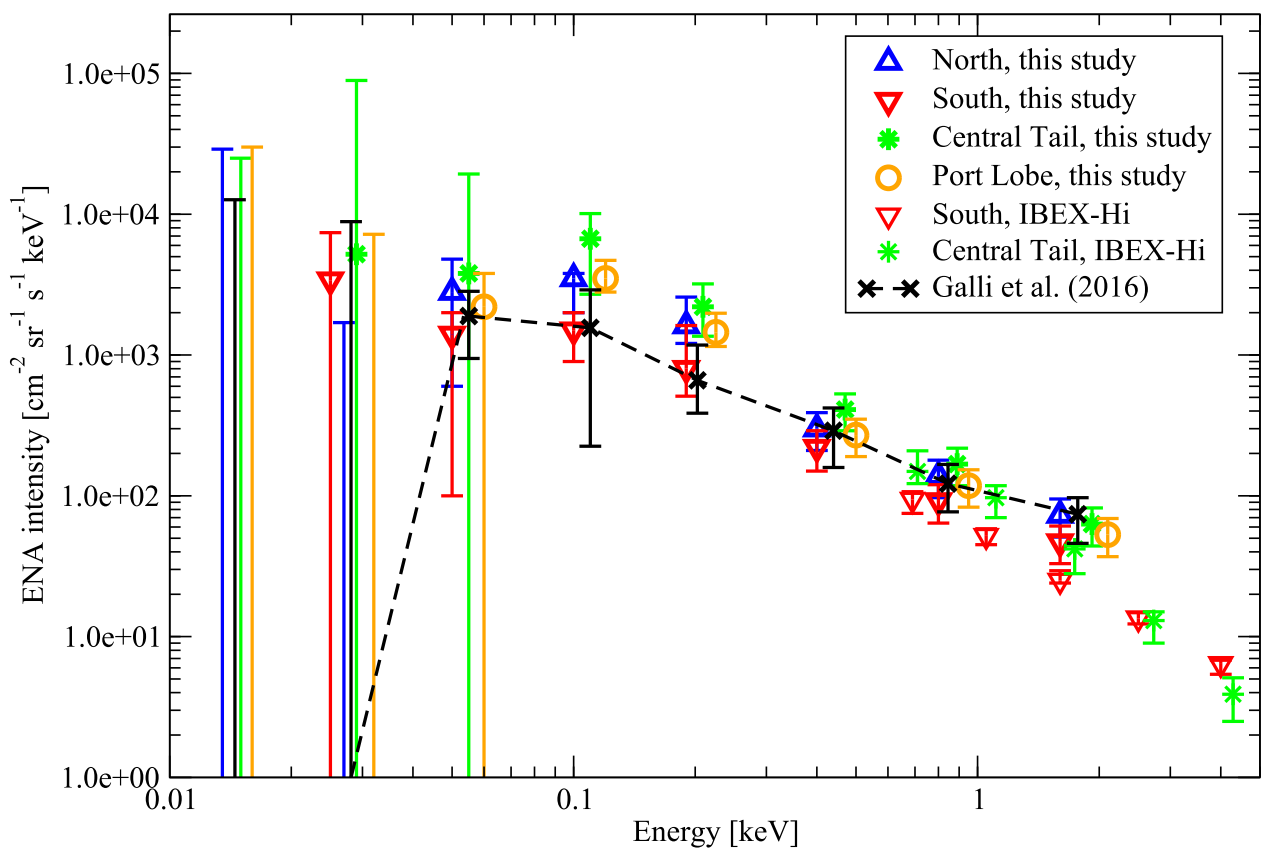

Figure 6. Energy spectrum of corrected ENA intensities for four geographic regions in the downwind hemisphere. "North": $\lambda=120^{\circ} \ldots 0^{\circ}, \beta=36^{\circ} \ldots 84^{\circ}$, "South": $\lambda=120^{\circ} \ldots 0^{\circ}, \beta=-84^{\circ} \ldots-36^{\circ}$, "Central Tail": $\lambda=120^{\circ} \ldots 24^{\circ}, \beta=-36^{\circ} \ldots+36^{\circ}$, "Port Lobe": $\lambda=24^{\circ} \ldots 312^{\circ}, \beta=-36^{\circ} \ldots+36^{\circ}$. Symbols in bold denote values derived from $I B E X$-Lo data. Thin symbols denote values from $I B E X$-Hi data. Black "x" symbols show the composite spectrum from a few smaller regions distributed over the downwind hemisphere (Galli et al. 2016). For improved clarity, some entries at identical energy were slightly shifted along the $x$-axis and two $I B E X$-Hi spectra were omitted from the plot.

corrected for Compton-Getting effect and survival probability. The energy range for which IBEX-Hi data are evaluated spans $\sim 0.5$ to $6 \mathrm{keV}$, with central energies at $0.71,1.11,1.74,2.73$, and $4.29 \mathrm{keV}$ (Funsten et al. 2009). Between 0.5 and $2.5 \mathrm{keV}$, the energy ranges of the two IBEX instruments thus overlap. We derived median values and $1 \sigma$ variability from the $I B E X-\mathrm{Hi}$
Data Release 10 over the four sky regions the same way as for IBEX-Lo data.

Figure 6 shows the energy spectra of the four regions; the values are the medians over single macropixels. The error bars indicate the $16 \%$ and $84 \%$ quantiles of included macropixel values or $30 \%$ relative error, whichever is larger. The four 
Table 3

Energy Spectrum of ENA Intensities

\begin{tabular}{|c|c|c|c|c|}
\hline $\begin{array}{l}\text { Central Energy } \\
(\mathrm{keV})\end{array}$ & North & South & $\begin{array}{r}\text { Central } \\
\text { Tail }\end{array}$ & $\begin{array}{l}\text { Port } \\
\text { Lobe }\end{array}$ \\
\hline 0.015 & $0_{-0}^{+29,000}$ & $\mathrm{~N} / \mathrm{A}$ & $0_{-0}^{+25,000}$ & $\overline{0_{-0}^{+30,000}}$ \\
\hline 0.029 & $0_{-0}^{+1700}$ & $3400_{-3400}^{+4000}$ & $5200_{-5200}^{+84,000}$ & $0_{-0}^{+7200}$ \\
\hline 0.055 & $2800_{-2200}^{+2000}$ & $1400_{-1300}^{+600}$ & $3800_{-3800}^{+15,500}$ & $2200_{-2200}^{+1600}$ \\
\hline 0.110 & $3500_{-1500}^{+300}$ & $1500_{-600}^{+500}$ & $6700_{-4000}^{+3400}$ & $3500_{-700}^{+1200}$ \\
\hline 0.209 & $1620_{-410}^{+960}$ & $800_{-290}^{+820}$ & $2200_{-840}^{+1000}$ & $1450_{-300}^{+530}$ \\
\hline 0.439 & $300 \pm 90$ & $220 \pm 70$ & $410 \pm 120$ & $270 \pm 80$ \\
\hline $0.872^{1}$ & $138 \pm 41$ & $92 \pm 28$ & $168 \pm 50$ & $118 \pm 35$ \\
\hline 1.821 & $73 \pm 22$ & $47 \pm 14$ & $63 \pm 19$ & $53 \pm 16$ \\
\hline
\end{tabular}

Note. Units are in $\mathrm{cm}^{-2} \mathrm{sr}^{-1} \mathrm{~s}^{-1} \mathrm{keV}^{-1}$. Data were sampled in the four downwind regions and averaged over all eight years of available IBEX-Lo observations.

energy spectra are compared with the median IBEX-Hi energy spectra sampled over the same four regions and with the previously published "Downwind spectrum" spectrum over the first four years of IBEX-Lo data (black " $\mathrm{x}$ " symbols, (Galli et al. 2016)). The colored symbols in bold are IBEX-Lo values, the colored thin symbols are $I B E X-H i$ values; blue triangles pointing up denote North, red triangles pointing down denote South, green asterisks denote the Central Tail, and orange circles denote the Port Lobe. The spectrum from Galli et al. (2016) was a composite of four smaller areas at the Central Tail and at the northern and southern flanks of the heliosheath $\left(\lambda=360^{\circ} \ldots 312^{\circ}\right)$, evaluated over the first four years of IBEXLo observations. It matches the seven-year downwind ENA spectra, but obviously blurs regional differences. Another point to note in this figure is that the spectral index of ENA intensities derived with $I B E X-\mathrm{Hi}$ around $1 \mathrm{keV}$ is steeper than for IBEX-Lo. This bend may be real, as ASPERA-3\&4 observations revealed a knee in the energy spectrum of heliospheric ENAs at $0.83 \pm 0.12 \mathrm{keV}$ (Galli et al. 2013). Table 3 lists the IBEX-Lo energy spectra illustrated in Figure 6.

\subsection{Temporal Trends}

If we want to compare the ENA signal from individual years to detect potential trends with time, we can do so only for the Port Lobe, where ram observations were available. The signalto-noise ratio of anti-ram observations for a single season proved insufficient (see Section 3). Moreover, some pixels were not sampled in every year. Figure 7 shows the time series of ENA intensities measured in the Port Lobe (upper panel) and the solar activity (lower panel). Only the eight seasons of ram observations (April-October) were included. Two temporal trends can be discerned in this figure. First, in the lowest two energy bins, the intensity drops in the years 2011-2013 to background levels before recovering in 2014-2016. This is probably ISN hydrogen responding to solar activity (see the discussion in Section 4.3). The second notable change occurs in the years 2014 to 2016, at intermediate energies from 0.05 to $0.2 \mathrm{keV}$. All other temporal variability remains within the error bars.

The North, South, and Central Tail regions could only be observed for anti-ram configuration; therefore, the error bars are generally too large to discern changes between single seasons for energies below $0.1 \mathrm{keV}$. At $0.11 \mathrm{keV}$, on the other hand, the last two of the eight seasons (October-April 2014/ 2015 and 2015/2016) significantly exceed the median values also in those three regions. Likewise, the four seasons from 2013-2016 feature significantly higher ENA intensities than for 2009-2012 in all three regions at $0.209 \mathrm{keV}$. The apparent dip of ENA intensity around $1 \mathrm{keV}$ in 2013 (purple and blue curves in Figure 7) agrees with contemporaneous IBEX-Hi (McComas et al. 2017) and INCA observations (Dialynas et al. 2017) of the downwind hemisphere. However, the ENA intensities measured with IBEX-Lo at $0.439,0.872$, and $1.821 \mathrm{keV}$ generally do not significantly change over the eight years in any of the four regions. Figure 7 is representative of the entire downwind hemisphere in this respect. The absence of significant year-to-year variations in solar wind energy ENAs ( 0.4 to $2.5 \mathrm{keV}$ ) is consistent with the finding of all previous studies of IBEX-Lo time-series (Fuselier et al. 2014; Galli et al. 2014; Reisenfeld et al. 2016). IBEX-Hi detected intensity variations with solar cycle on the order of $30 \%$ or less around $1 \mathrm{keV}$ (McComas et al. 2017). Such variations usually cannot be distinguished with IBEX-Lo because of the poorer signalto-noise ratio (Galli et al. 2014).

If the observed increase in ENA intensity were to occur everywhere between 2012 June and 2013 January, we might suspect an instrumental effect, as the post-acceleration bias in $I B E X$-Lo changed in that period (see Section 2). However, because the intensity increases only in 2014 June or 2015 January in several cases, and because the intensities at higher energies do not rise at all in later years, a natural cause seems more plausible. If indeed more ENAs at intermediate energies were produced in the heliosheath at some time during the solar cycle, we must first derive the trace-back time of the detected ENAs. Because temporal variation is likely correlated with the solar cycle, the trace-back time corresponds to the time span between the emission of the source plasma (the solar wind) from the Sun, its transit to the heliosheath where it becomes ENAs, and the transit time back to the inner heliosphere, where the ENAs are detected at IBEX. Following the notation by Reisenfeld et al. (2012, 2016),

$$
t_{\mathrm{tb}}(E)=\frac{d_{\mathrm{TS}}}{v_{\mathrm{sw}}}+\frac{l_{\mathrm{IHS}} / 2}{v_{\mathrm{ms}}}+\frac{d_{\mathrm{TS}}+l_{\mathrm{IHS}} / 2}{v_{\mathrm{ENA}}(E)},
$$

$t_{\mathrm{tb}}(E)$ is the trace-back time for ENAs of energy $E$ originating in the inner heliosheath, $d_{\mathrm{TS}}$ is the distance to the termination shock, $l_{\mathrm{IHS}}$ is the thickness of the inner heliosheath, $v_{\mathrm{sw}}$ is the solar wind proton speed, $v_{\mathrm{ms}}$ is the average magnetosonic speed in the inner heliosheath, and $v_{\mathrm{ENA}}(E)$ is the speed of an ENA of energy $E$ observed at IBEX. Reisenfeld et al. (2016) estimated $d_{\mathrm{TS}} \approx 130 \mathrm{au}$ and $l_{\mathrm{IHS}} \approx 210 \mathrm{au}$ for the north pole, $d_{\mathrm{TS}} \approx 110 \mathrm{au}$ and $l_{\mathrm{IHS}} \approx 160 \mathrm{au}$ for the south pole, and $v_{\mathrm{sw}}=690 \mathrm{~km} \mathrm{~s}^{-1}$ for the polar regions. We assigned $l_{\mathrm{IHS}}=280 \mathrm{au}$ and $d_{\mathrm{TS}}=110 \mathrm{au}$ to the Central Tail and $l_{\mathrm{IHS}}=180 \mathrm{au}$ and $d_{\mathrm{TS}}=120 \mathrm{au}$ to the Port Lobe (see next Section 5). The magnetosonic speed in the inner heliosheath (Reisenfeld et al. 2012),

$$
\sqrt{v_{s}^{2}+v_{A}^{2}} \approx \sqrt{\frac{5}{3} \frac{P}{\rho}} \approx 430 \mathrm{~km} \mathrm{~s}^{-1}
$$

does not change with latitude if a similar plasma pressure $(0.12$ $\mathrm{pPa}=1.2$ pdyn $\left.\mathrm{cm}^{-2}\right)$ and proton density $\left(640 \mathrm{~m}^{-3}\right)$ are assumed for all downwind directions. Toward Central Tail and Port Lobe, the solar wind speed inside the termination shock is assumed to be $440 \mathrm{~km} \mathrm{~s}^{-1}$ (Whang 1998). With these speeds 

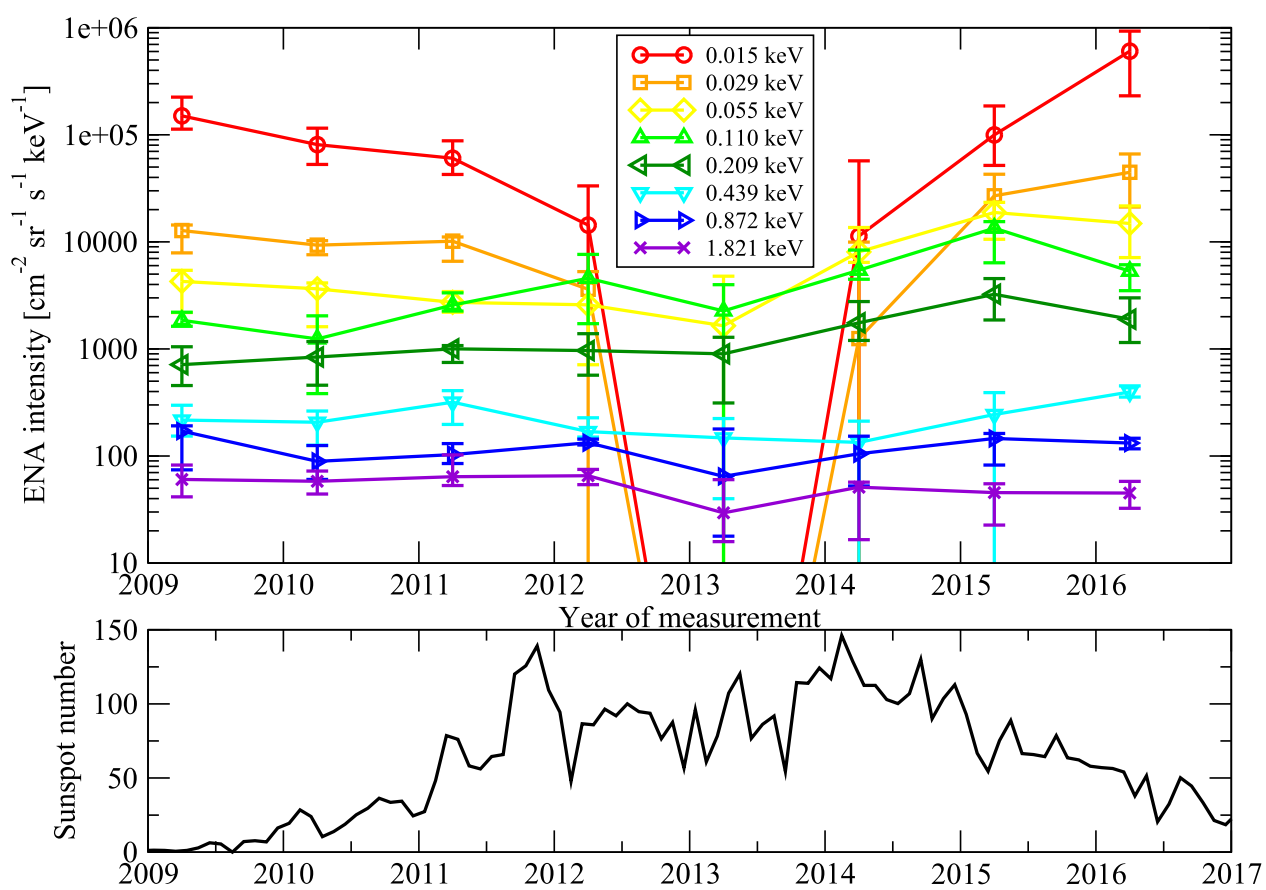

Figure 7. Time series of measured ENA intensities vs. solar activity. Top panel: rainbow-colored sequence from red (energy bin $1,0.015 \mathrm{keV}$ ) to indigo (energy bin 8 , $1.821 \mathrm{keV}$ ) of ENA intensities measured in the Port Lobe region for individual seasons. Only the eight ram seasons, with their smaller error bars, are shown. Lower panel: monthly averaged sunspot number (SILSO, World Data Center 2008).

and distances, Equation (2) yields the trace-back times shown in Table 4 for the five cases we need to distinguish. The effects of solar radiation pressure and gravity on the travel time of lowenergy ENAs were considered. The uncertainties stated in Table 4 are introduced by the radiation pressure changing with solar activity.

Two things should be noted from this table of trace-back times. First, trace-back dates for ENAs sampled at $15 \mathrm{eV}$ (the lowest energy bin) from the anti-ram direction in the ecliptic plane (Central Tail or Port Lobe) differ by several years, depending on whether they were sampled during solar minimum (2009) or solar maximum conditions (2014). The incoming ENAs at these low energies are mixed together over an entire solar cycle, so the concept of a trace-back time characteristic for a given year and region in the sky cannot distinguish between solar wind emitted during solar maximum and solar maximum. Second, time series of ram and anti-ram measurements at low energies in the ecliptic plane cannot be directly compared because their corrected energies, and thus trace-back times, are grossly different. This is another reason why we show only the ram measurements from the Port Lobe region in Figures 7 and 8.

The upper panel in Figure 8 shows the time-series of ENA intensities measured at 0.11 (dashed lines) and $0.209 \mathrm{keV}$ (dashed-dotted lines) for all four downwind regions versus trace-back date. Orange symbols denote Port Lobe (ram observations only), blue symbols denote North, red symbols denote South, green symbols denote Central Tail (only anti-ram observations available). The lower panel of Figure 8 illustrates the solar activity via the monthly mean sunspot number (SILSO, World Data Center 2008). The heliospheric ENA production seems to be anti-correlated with solar activity in the North, South, and Port lobe region of the downwind hemisphere. A simple regression analysis reveals that, for seven out of eight time series in Figure 8, the ENA intensity significantly
Table 4

Trace-back Times in Years

\begin{tabular}{lccccc}
\hline $\begin{array}{l}\text { Central energy } \\
(\mathrm{keV})\end{array}$ & North & South & $\begin{array}{c}\text { Port Lobe, } \\
\text { ram }\end{array}$ & $\begin{array}{c}\text { Port Lobe, } \\
\text { anti-ram }\end{array}$ & $\begin{array}{c}\text { Central Tail, } \\
\text { anti-ram }\end{array}$ \\
\hline 0.015 & $20 \pm 1$ & $16 \pm 1$ & $14 \pm 1$ & $36 \pm 5$ & $43 \pm 7$ \\
0.029 & $15 \pm 1$ & $12 \pm 1$ & $12 \pm 1$ & $23 \pm 1$ & $27 \pm 2$ \\
0.055 & 12 & 10 & 9.3 & 15 & 18 \\
0.110 & 10 & 8.0 & 7.9 & 11 & 13 \\
0.209 & 7.8 & 6.2 & 6.6 & 8.2 & 9.7 \\
0.439 & 6.0 & 4.8 & 5.4 & 6.1 & 7.2 \\
0.872 & 4.8 & 3.9 & 4.6 & 4.9 & 5.8 \\
1.821 & 4.0 & 3.2 & 3.9 & 4.1 & 4.8 \\
\hline
\end{tabular}

Note. Data are from ENAs measured in IBEX-Lo energy bins for the five cases relevant to this study.

(confidence levels between 2 and 6 sigma) increases with time from trace-back dates 1999-2003 (the previous solar maximum) until the end of series at 2006-2010 (solar minimum). The time series for $0.1 \mathrm{keV}$ at Central Tail does not follow this trend. Toward the North, the ENA increase set in at a traceback date of 2005; toward the South and Port Lobe, the ENA increase followed somewhat later in 2006-2007. From the anticorrelation of ENA intensity and solar activity, we predict that the ENA intensities at $0.2 \mathrm{keV}$ will remain high until the middle of 2017, then will decrease again when the trace-back date corresponds to increasing solar activity. The decrease of $0.1 \mathrm{keV}$ ENA intensity should follow one to two years later. No conclusion can be reached yet with respect to the time variability of the Central Tail. The ENA intensities at $0.1 \mathrm{keV}$ (green dashed line) linked to a trace-back date before 1999 show no tendency to increase with low solar activity.

At 0.4 and $0.9 \mathrm{keV}$, only one out of the eight time-series from the downwind regions exhibits a slope significantly 

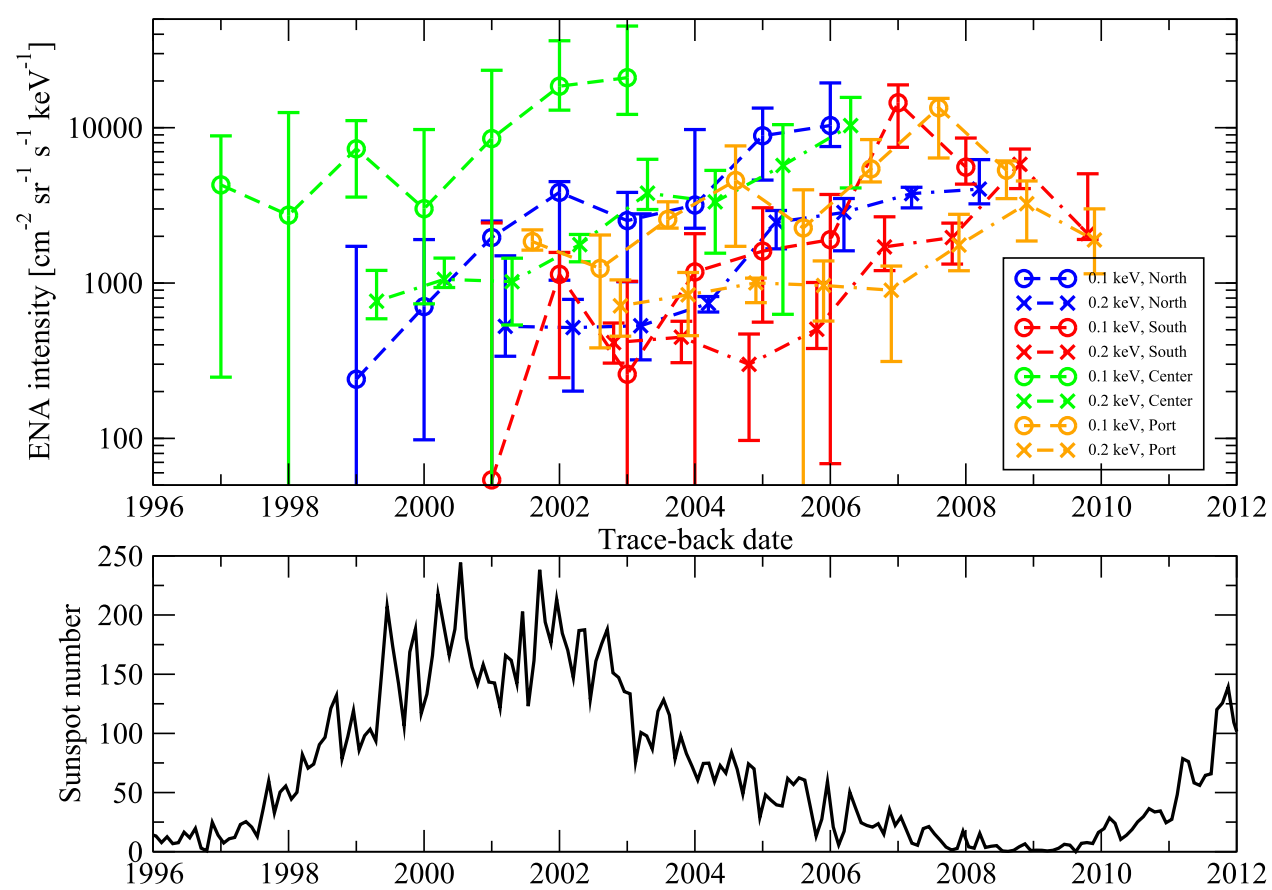

Figure 8. Anti-correlation of heliospheric ENAs at 0.1 and $0.2 \mathrm{keV}$ with solar activity. The upper panel shows the time-series of ENA intensities for all four downwind regions (colored curves) vs. trace-back date, the lower panel shows the monthly mean sunspot number as a proxy for solar activity (SILSO, World Data Center 2008).

different from zero. In all other cases, a constant fits all eight seasonal values within respective error bars. The variations of ENA intensity with time are generally less pronounced at solar wind energies than for $0.08-0.3 \mathrm{keV}$ and $3-6 \mathrm{keV}$ (see McComas et al. (2017) regarding the temporal evolution over the full sky and Reisenfeld et al. (2016) for the polar regions). With eight years of observations available, both IBEX-Lo and $I B E X$-Hi can be used to track the imprint of the solar cycle on the heliosheath plasma.

We cannot answer yet which physical process causes the anti-correlation between solar activity and production of 0.1-0.2 keV ENAs in the heliosheath. However, we have seen that the temporal evolution of ENA intensities looks similar for all directions in the downwind hemisphere, with the possible exception of the Central Tail. This implies via the trace-back time in Equation (2) that the region of ENA production has a similar distance toward the poles and toward the flanks of the heliotail. The estimate for the Central Tail may differ from our assumption. In Section 5, we will investigate these assumptions by deriving the heliosheath thickness from the total plasma pressure averaged over time. Once the IBEX-Lo time-series cover an entire solar cycle, we can use an updated version of Figure 8 to optimize the trace-back times-and from there, the travel distance of ENAs from the downwind hemisphere.

\subsection{Interstellar Neutral Hydrogen Observed at the Lowest-energy Bins}

In the overview figure of energy bin 1 (Figure 1), the ISN inflow was seen to extend to longitudes $>300^{\circ}$ in the ecliptic plane. Looking at the temporal evolution in Figure 7 for the Port Lobe, we recognize the ISN signal in energy bin 1 in 2009-2011, then it vanishes in 2012, only to reappear in 2014. Remember that those ENA measurements include only the ram observations. The anti-ram measurements of the same sky direction usually yield no detectable ENA signal (see the black macropixels at $15 \mathrm{eV}$ and $29 \mathrm{eV}$ in Figure 3). Heliospheric ENAs of $15 \mathrm{eV}$ with trace-back times of decades obviously cannot produce a bi-annual intensity change, so we conclude that this is indeed the outermost part of the seasonal ISN inflow.

The energy of ISN hydrogen is too low to produce any signal above energy bin 2, and ISN helium produces a strong signal in $I B E X$-Lo in all four energy bins below $150 \mathrm{eV}$ (Saul et al. 2013). This can be easily understood from the energy of the ISN particles entering IBEX-Lo. Whereas ISN helium has a maximum relative energy of $130 \mathrm{eV}$ (Galli et al. 2015) for ram measurements, ISN hydrogen will have, at most, the same velocity as ISN helium; however, having four times less mass, it can only reach $33 \mathrm{eV}$. This is close to the central energy of bin 2 at $29 \mathrm{eV}$. Any non-gravitational influence on the ISN hydrogen trajectory, such as solar radiation pressure, will tend to further slow down hydrogen with respect to helium. As the signal temporarily disappears only in energy bins 1 and 2-but not in energy bins 3 and 4-in Figure 7, we conclude that this signal is mostly ISN hydrogen. In the ecliptic plane, it extends to an apparent direction of $336^{\circ} \pm 6^{\circ}$, which is even wider than the Warm Breeze of the secondary helium (Kubiak et al. 2016). The latter blends into background around $300^{\circ} \pm 6^{\circ}$, judging from count rate maps in analogy to Figure 1 for $0.055 \mathrm{keV}$.

The variation of the ISN hydrogen signal from 2009 to 2016 in Figure 7 is probably caused by the varying survival probability for neutral $\mathrm{H}$ reaching the inner solar system depending on solar cycle. Saul et al. (2013) found that this hypothesis explained the IBEX-Lo observations of ISN hydrogen during the first part of the solar cycle. The rapid recovery of the ISN hydrogen in 2014 is puzzling, however. Based on solar activity (see Figure 7), we would have expected the signal to recover only in 2016. It is possible that the model used to estimate hydrogen ENA losses due to re-ionization close to $1 \mathrm{au}$ is not accurate enough at these very low energies. 
We cannot verify whether the heliospheric ENAs are affected the same way, i.e., if they also rise rapidly in 2014 in all other downwind regions at $15 \mathrm{eV}$ and $29 \mathrm{eV}$. At these energies, the anti-ram observations do not allow for a meaningful seasonal analysis. We will devote a future study to this ISN hydrogen signal and how it expands and diminishes over a full solar cycle. For the discussion of heliospheric ENAs in the following section, we will disregard the ISN contribution to the Port Lobe below $0.055 \mathrm{keV}$.

\section{Implications for the Heliosheath in the Downwind Direction}

Throughout this discussion, we assume that the observed ENAs originated exclusively in the inner heliosheath. Contributions from the outer heliosheath are unlikely because of the increasing heliosheath thickness in the downwind direction. The ENA intensities observed with IBEX-Lo from the Voyager 1 and Voyager 2 directions in the upwind hemisphere can be reproduced without a contribution of ENAs from the outer heliosheath (Galli et al. 2016). It is therefore unlikely that ENAs from the outer heliosheath contribute notably to the ENA intensity in the downwind hemisphere.

The ENA measurements provide insight into the integrated plasma pressure over the line-of-sight thickness of the source plasma population from which the ENAs are emitted. We repeat the plasma pressure calculation presented by Schwadron et al. (2011) (also see Fuselier et al. 2012; Reisenfeld et al. 2012, 2016; Schwadron et al. 2014; Galli et al. 2016) for the new ENA energy spectra averaged over the four regions. Because this is based on observations, the integration is done step-wise over each of the energy bins of the instrument; the reference frame of the plasma pressure is heliocentric, i.e., does not move with the plasma bulk flow speed $u_{R}$ (Schwadron et al. 2011):

$$
\Delta P \times l=\frac{2 \pi m^{2}}{3 n_{H}} \frac{\Delta E}{E} \frac{j_{\mathrm{ENA}}}{\sigma(E)} \frac{\left(v_{\mathrm{ENA}}+u_{R}\right)^{4}}{v_{\mathrm{ENA}}} .
$$

The measured intensity $j_{\mathrm{ENA}}$ of neutralized hydrogen at a given energy thus translates into the product $\Delta P \times l$ of the pressure of the parent ion population in the heliosheath that is the ENA emission source and the thickness of this ion population source region along the instrument line of sight. This pressure includes only the internal pressure of the moving plasma; there is no ram-pressure term contributing to the balance for the downwind hemisphere. Equation (4) states that the product of pressure times ENA emission thickness can be derived from observations, but further assumptions will be needed to separately obtain the two values. The equation can be rewritten in the notation of Fuselier et al. (2012) as the product of a stationary pressure (the internal pressure in the inertial reference frame with $u_{R}=0$ ) times a correction factor for the plasma bulk flow velocity with respect to the heliocentric rest frame:

$$
\begin{gathered}
\Delta P \times l=\frac{4 \pi m_{H}}{3 n_{H}} \frac{v_{\mathrm{ENA}} j_{\mathrm{ENA}}\left(E_{0}\right)}{\sigma\left(E_{0}\right)} \int_{E_{0}-\Delta E / 2}^{E_{0}+\Delta E / 2} d E\left(\frac{E}{E_{0}}\right)^{-\gamma} c_{f} \\
c_{f}=\frac{\left(v_{\mathrm{ENA}}+u_{R}\right)^{4}}{v_{\mathrm{ENA}}^{4}} .
\end{gathered}
$$

In Equation (5), $\Delta E$ denotes the width of the respective energy bin and $\gamma$ is the spectral index (see Equation (1)). For the typical radial velocity of solar wind in the downwind hemisphere of the inner heliosheath, we assumed $u_{R}=140 \mathrm{~km}$ $\mathrm{s}^{-1}$ everywhere, as measured by Voyager 2 (Whang et al. 1999; Schwadron et al. 2011) and similar to the range of 100-150 km $\mathrm{s}^{-1}$ assumed by Zirnstein et al. (2016a) over the first $100 \mathrm{au}$ beyond the termination shock. More precisely, $u_{R}$ would be a function of heliolatitude with faster plasma speeds-up to 225 $\mathrm{km} \mathrm{s}^{-1}$ _occurring toward the poles (Reisenfeld et al. 2016). Such a latitudinal dependence could be formulated if we assumed a shock jump of 2.5 everywhere (Schwadron et al. 2011). For this discussion, however, let us assume a constant plasma bulk flow speed for the entire downwind hemisphere. As in previous $I B E X$-related papers on pressure in the heliosheath, let us first assume a constant density of neutral hydrogen everywhere in the inner heliosheath with $n_{\mathrm{H}}=0.1$ $\mathrm{cm}^{-3}$ (Schwadron et al. 2011; Gloeckler \& Fisk 2015). We will re-assess this assumption at the end of this section. The chargeexchange cross section $\sigma\left(E_{0}\right)$ between protons and neutral hydrogen is taken from Lindsay \& Stebbings (2005), decreasing from 4 to $2 \times 10^{-15} \mathrm{~cm}^{-2}$ as the ENA energy increases from 0.015 to $1.821 \mathrm{keV}$. We applied Equation (5) to the average ENA intensities in Figure 6 and Table 3 to calculate stationary and dynamic pressure for all regions. In the stationary case, the ENAs at solar wind energies would dominate the total pressure balance. For the following discussions and illustrations, however, we will concentrate on the dynamic pressure because the plasma is flowing away from the Sun.

\subsection{Plasma Pressure in the Heliosheath}

To obtain the full plasma pressure $P$ in the heliosheath we would like to integrate the ENA spectrum from zero to infinite energy. As will be shown briefly hereafter, IBEX-Lo usually covers most energies at the lower end that contribute to the total pressure. To assess how much pressure would be added at energies above the IBEX-Lo cutoff at $2.5 \mathrm{keV}$, we also considered the $I B E X$-Hi spectra averaged over the four regions (see Table 3). As in our previous study (Galli et al. 2016), the relative uncertainty attributed to $I B E X-\mathrm{Hi}$ measurements is either $20 \%$ or the standard deviation between the four different regions, whichever is larger. Whereas the spectral index derived from $I B E X$-Hi spectra is steeper than for IBEX-Lo spectra at the overlapping energy, the dynamic pressure added over the two IBEX-Lo bins 7 and 8 (covering roughly 0.6-2.5 $\mathrm{keV}$ ), agrees well with the dynamic pressure integrated over IBEX-Hi energy bins 2 to $4(0.5-2.5 \mathrm{keV})$ : (72 and 67) pdyn $\mathrm{cm}^{-2}$ au for the North, (47 and 40) pdyn $\mathrm{cm}^{-2}$ au for the South, (74 and 78) pdyn $\mathrm{cm}^{-2}$ au for the Central Tail, and (57 and 50) pdyn $\mathrm{cm}^{-2}$ au for the Port Lobe. We therefore combined the lower six energy bins of IBEX-Lo with the upper five energy bins of $I B E X-\mathrm{Hi}$ to obtain four composite energy spectra spanning the whole range from 0.01 to $6 \mathrm{keV}$. These pressure spectra are averages over all eight (IBEX-Lo) or seven (IBEX$\mathrm{Hi}$ ) years of observations. The resulting $\Delta P \times l$ per individual energy bin is shown for all four regions in Figure 9 (orange triangles pointing up indicate North; green triangles pointing down indicate South; red asterisks represent the Central Tail; 


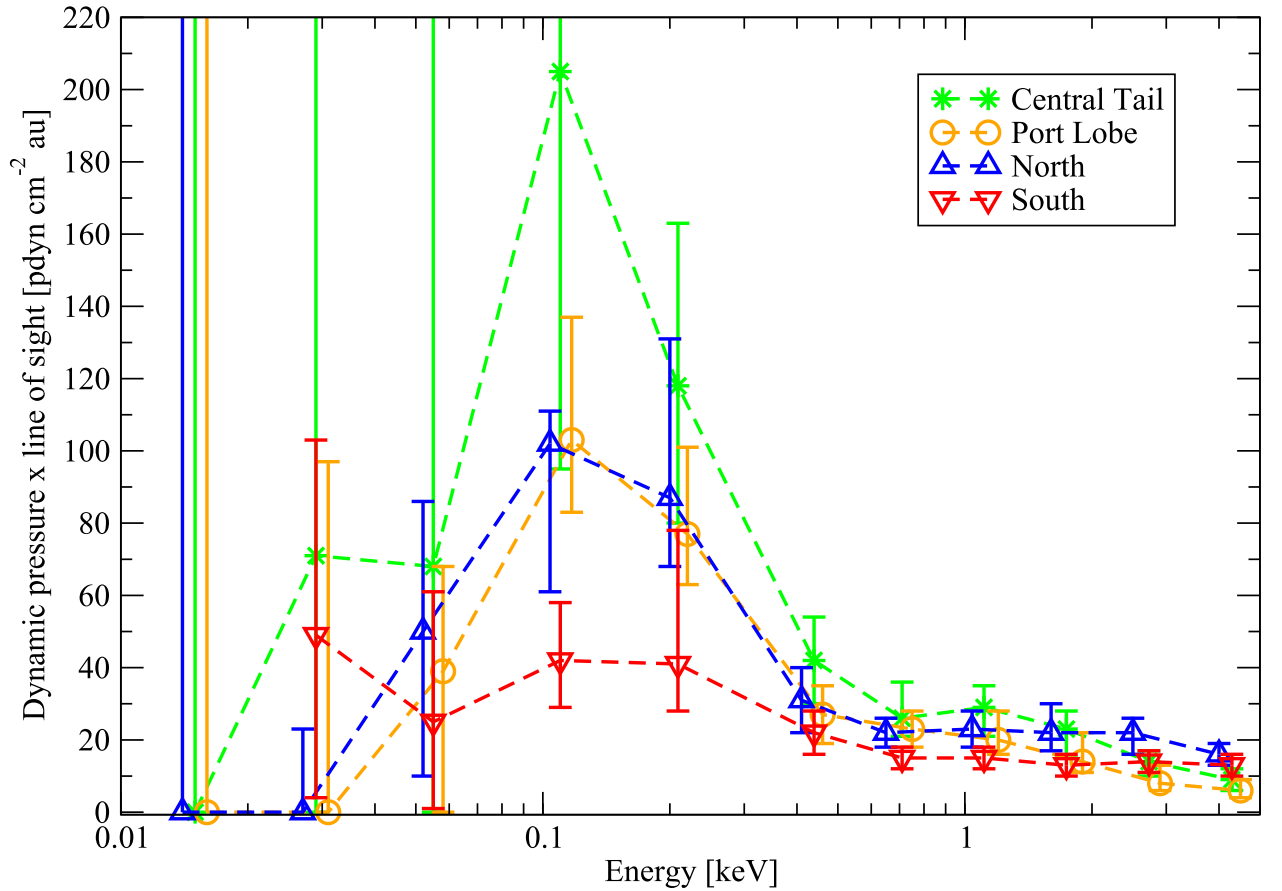

Figure 9. Energy spectrum of $\Delta P \times l$ (partial dynamic pressure times ENA emission thickness) in units of pdyn $\mathrm{cm}^{-2}$ au. The symbols have the same meaning as in Figure 6, but here $I B E X$-Lo and $I B E X$-Hi data have been combined into composite spectra from the six lower-energy bins of $I B E X$-Lo and the five higher-energy bins of $I B E X$-Hi.

blue circles represent the Port Lobe). The error bars represent the variability within a region; the uncertainties of the plasma bulk flow velocity were neglected.

The values of the pressure spectra plotted in Figure 9 are stated in Table 5. Table 6 gives the corresponding total $P \times l$ over two energy ranges ( 0.01 to $6 \mathrm{keV}$ and 0.08 to $6 \mathrm{keV}$ ). The ENA energies at $0.11 \mathrm{keV}$ and $0.209 \mathrm{keV}$ contribute the most to the total pressure for all four downwind regions. We therefore set $150 \mathrm{eV}$ as the relevant energy to discuss the cooling length (Schwadron et al. 2011, 2014) in the downwind heliosheath. The energies below the roll-over around $100 \mathrm{eV}$ contribute little, on average, to the total pressure because most macropixels feature no discernible ENA signal above the background (see Figure 3). However, because of the large upper error bars and the large correction factor at low ENA energies $\left(c_{f}=170\right.$ at $\left.15 \mathrm{eV}\right)$, the Central Tail could feature a high upper limit of 1800 pdyn $\mathrm{cm}^{-2}$ au if the energy range were extended down to $10 \mathrm{eV}$. We therefore added the plasma pressure over two different energy ranges in Table 6 to indicate the uncertainties at the lowest ENA energies.

Before interpreting the derived values of $P \times l$, we would like to caution the reader about the effect of solar activity on the values in Table 6. That table incorporates all available observations, thus blending ENA observations representative of high and low solar activity conditions in the heliosheath. Because IBEX observations do not yet cover an entire solar cycle, we cannot create separate plasma pressure spectra for low and high solar activity over the entire energy range of $I B E X$-Lo and IBEX-Hi. The trends of ENA intensities at 0.1 and $0.2 \mathrm{keV}$ in Figure 7 indicate that the $\Delta P \times l$ decreases by a factor of three during high solar activity, with respect to the average stated in Table 6 , and increases by a similar factor for low solar activity conditions. Because ENAs of these energies are a dominant contribution to the total plasma pressure (see Figure 9), $P \times l$ varies strongly between the two extreme cases:
Table 5

Dynamic Pressure Times ENA Emission Thickness $\Delta P \times l$

\begin{tabular}{lcrcrr}
\hline \hline $\begin{array}{l}\text { Central energy } \\
(\mathrm{keV})\end{array}$ & $\begin{array}{c}\text { Correction } \\
\text { factor }\end{array}$ & North & South & $\begin{array}{r}\text { Central } \\
\text { Tail } \\
\mathrm{cm}^{-2}\end{array}$ & $\begin{array}{r}\text { Port } \\
\text { Lobe } \\
\text { au) }\end{array}$ \\
\hline 0.015 & 170 & $0_{-0}^{+317}$ & $\mathrm{~N} / \mathrm{A}$ & $0_{-0}^{+267}$ & $0_{-0}^{+327}$ \\
0.029 & 69 & $0_{-0}^{+23}$ & $49_{-45}^{+54}$ & $71_{-71}^{+1124}$ & $0_{-0}^{+97}$ \\
0.055 & 31 & $50_{-40}^{+36}$ & $25_{-24}^{+36}$ & $68_{-68}^{+281}$ & $39_{-39}^{+29}$ \\
0.110 & 15 & $102_{-41}^{+9}$ & $42_{-13}^{+16}$ & $205_{-110}^{+95}$ & $103_{-20}^{+34}$ \\
0.209 & 8.3 & $87_{-19}^{+44}$ & $41_{-13}^{+37}$ & $118_{-38}^{+45}$ & $77_{-14}^{+24}$ \\
0.439 & 4.8 & $31_{-9}^{+9}$ & $22_{-6}^{+6}$ & $42_{-12}^{+12}$ & $27_{-8}^{+8}$ \\
0.71 & 3.6 & $22_{-4}^{+4}$ & $15_{-3}^{+3}$ & $26_{-5}^{+10}$ & $23_{-5}^{+5}$ \\
1.11 & 2.9 & $23_{-5}^{+5}$ & $15_{-3}^{+3}$ & $29_{-8}^{+6}$ & $20_{-4}^{+8}$ \\
1.74 & 2.4 & $22_{-5}^{+8}$ & $13_{-3}^{+3}$ & $23_{-8}^{+5}$ & $14_{-3}^{+8}$ \\
2.73 & 2.0 & $22_{-6}^{+4}$ & $14_{-3}^{+3}$ & $14_{-4}^{+3}$ & $8_{-2}^{+5}$ \\
4.29 & 1.8 & $16_{-3}^{+3}$ & $13_{-3}^{+3}$ & $9_{-3}^{+3}$ & $6_{-2}^{+3}$ \\
\hline
\end{tabular}

Note. As defined in Equation (5). Derived from the composite IBEX-Lo (taken from Table 3) and IBEX-Hi (McComas et al. 2017) spectra for the four downwind Regions for all eight available years (seven years in the case of $I B E X$-Hi). For the correction factor $c_{f}$ in the second column (Equation (6)), a constant $140 \mathrm{~km} \mathrm{~s}^{-1}$ was assumed everywhere.

Table 6

Integral Dynamic Pressures Times ENA Emission Thickness

\begin{tabular}{|c|c|c|c|c|c|}
\hline $\begin{array}{l}\text { Energy range } \\
(\mathrm{keV})\end{array}$ & $\begin{array}{l}\text { Observation } \\
\text { time }\end{array}$ & $\begin{array}{l}\text { North } \\
P \times l\end{array}$ & $\begin{array}{l}\text { South } \\
\text { (pdyn }\end{array}$ & $\begin{array}{r}\text { Central } \\
\text { Tail } \\
\mathrm{cm}^{-2}\end{array}$ & $\begin{array}{r}\text { Port } \\
\text { Lobe } \\
\text { au) }\end{array}$ \\
\hline $0.01-6.0$ & 2009-2016 & $374_{-62}^{+323}$ & $248_{-55}^{+77}$ & $605_{-153}^{+1194}$ & $318_{-47}^{+345}$ \\
\hline $0.08-6.0$ & 2009-2016 & $325_{-47}^{+47}$ & $174_{-21}^{+42}$ & $466_{-118}^{+107}$ & $279_{-26}^{+45}$ \\
\hline $0.01-6.0$ & 2009-2011 & 210 & 150 & 280 & 180 \\
\hline
\end{tabular}

Note. The upper two lines are the sums from Table 5, the bottom line (bold) represents the lower limits for high solar activity conditions and best signal-to-noise ratio. 
we estimate that $P \times l$ increases from $300 \pm 100$ to $1050 \pm 300$ pdyn $\mathrm{cm}^{-2}$ au in the Central Tail (integrated from $0.08-6 \mathrm{keV}$ ) as the heliosheath changes from high to low solar activity conditions. The IBEX-Lo observations of 2017 and 2018 will elucidate whether this anti-correlation with solar activity is real: these years combine a low present solar activity (which allows for a better signal-to-noise ratio) with a traceback time for $0.1 \mathrm{keV}$ ENAs corresponding to the previous solar minimum. However, we can confidently state the lower limits of $P \times l$ over the solar cycle. We assumed the lower limits in Table 6 and reduced the contributions at 0.1 and $0.2 \mathrm{keV}$ according to the temporal trend in Figure 7. The results are representative of high solar activity conditions and optimum signal-to-noise ratio during the first three years of IBEX-Lo observations. These lower limits, evaluated from 0.01 to $6 \mathrm{keV}$, read: $210,150,280$, and 180 pdyn $\mathrm{cm}^{-2}$ au for the North, South, Central Tail, and Port Lobe, respectively.

The main challenge with interpreting the product $P \times l$ is disentangling the two factors. If they are independent-and thus separable - a governing assumption is that $P$ is constant over $l$. Both parameters are unknown a priori, as no in situ measurements are available, contrary to the upwind hemisphere observed by the Voyager spacecraft (Decker et al. 2005; Burlaga et al. 2008; Gurnett et al. 2013; Stone et al. 2013). If the plasma pressure is assumed to be similar over all directions in the heliosheath, the numbers in Table 5 translate directly into the thickness of the emission structures along lines of sight in the heliosheath. But what should that total pressure be?

Most studies, irrespective of whether they are based on models or observations, predict a total plasma pressure of 1-2 pdyn $\mathrm{cm}^{-2}$ in the inner heliosheath: Livadiotis et al. (2013) derived a total pressure of 2.1 pdyn $\mathrm{cm}^{-2}$ over the entire energy spectrum, comparing the expected plasma pressure from a kappa distribution of protons with the plasma pressure derived from $I B E X$-Hi energy spectra. These authors found the same value for all sky directions except for the ENA Ribbon toward the nose of the heliosphere. Rather at the lower limit, Reisenfeld et al. (2012) and Gloeckler \& Fisk (2011) derived a total plasma pressure of 1.2 pdyn $\mathrm{cm}^{-2}$ from Voyager and early IBEX observations. This pressure is dominated by the heliospheric pickup ions, which contribute $1.0 \pm 0.5$ pdyn $\mathrm{cm}^{-2}$ (Gloeckler \& Fisk 2011). Galli et al. (2016) derived 1.4 pdyn $\mathrm{cm}^{-2}$ for a few regions in the flanks and in the heliotail for the IBEX-Lo energy range from $10 \mathrm{eV}$ to $2.5 \mathrm{keV}$. Reisenfeld et al. (2016) derived a heliosheath thickness of $160 \mathrm{au}$ for the south pole region and 210 au for the north pole region, using a different approach of analyzing the temporal variability of heliospheric ENAs at several $\mathrm{keV}$. With our values for $P \times l$ in Table 6, these dimension imply $P=1.8+1.6-0.3$ pdyn $\mathrm{cm}^{-2}$ from 0.01 to $6 \mathrm{keV}$ to the North, which equals the $1.6 \pm 0.4$ pdyn $\mathrm{cm}^{-2}$ to the South. This reduces to $P=1.3 \pm 0.2$ pdyn $\mathrm{cm}^{-2}$ from 0.08 to $6 \mathrm{keV}$ for the two polar regions. Applying $P=1.3 \mathrm{pdyn}^{-2}$ to Table 5, we then find $l=215+35-22$ au for the ENA emission thickness toward the Port Lobe and $360 \pm 85$ au toward the Central Tail. The lower limits of $P \times l=210$ and 150 pdyn $\mathrm{cm}^{-2}$ au imply $P=1.0$ pdyn $\mathrm{cm}^{-2}$; hence, the heliosheath must be at least $280 \mathrm{au}$ in the Central Tail and $180 \mathrm{au}$ toward the Port Lobe for any time during the solar cycle.

The difference of $P \times l$ observed toward North and South confirms the dichotomy found by Reisenfeld et al. (2016) at higher energies $(0.5$ to $6 \mathrm{keV})$. This independent confirmation at lower energy indicates that the downwind heliosheath likely extends farther to the north than to the south. The dichotomy already appears in the stationary pressure; the lines of sight would be similar at both poles only if the radial bulk flow velocity toward the south were much higher $\left(u_{R} \approx 200 \mathrm{~km} \mathrm{~s}^{-1}\right.$ instead of the assumed $140 \mathrm{~km} \mathrm{~s}^{-1}$ ) than toward the north to compensate for the observed two-fold difference via the dynamic correction factor (see Equation (6)).

The minimum ENA emission thickness in the Central Tail region considerably exceeds the lower limits encountered for the other downwind regions (high latitudes or the flank of the heliosheath around $\lambda=0^{\circ}$ ). The presumed tail of the heliosheath therefore is not visibly deflected from the nominal downwind direction of $76^{\circ}$ ecliptic longitude (McComas et al. 2015). This confirms Ly $\alpha$ observations by Wood et al. (2014) of interstellar absorption toward nearby stars in the downwind direction. The Port and Starboard Tail lobes show up beside the nominal downwind direction in ENA maps above $1.5 \mathrm{keV}$ energy, but these lobes then seem to blend into the globally distributed ENA flux at solar wind energy (McComas et al. 2013; Zirnstein et al. 2016a). A similar bilobate structure at lower energies has not been detected so far. Because the total plasma pressure is dominated by those lower energies, the $I B E X$ data are consistent with a symmetric heliotail not notably offset from the nominal downwind direction.

\subsection{Plasma Cooling Length and the Dimension of the Heliosheath}

Schwadron et al. (2011) introduced the concept of a cooling length $l_{\text {cool }}$ in the inner heliosheath as the timescale for plasma ions being neutralized times the plasma bulk flow velocity:

$$
l_{\mathrm{cool}}=\frac{u_{R}}{n_{\mathrm{H}} \sigma v_{\mathrm{ENA}}}
$$

The mean free path length a hydrogen ENA of the same energy can travel before being lost to re-ionization is much longer than that. Schwadron et al. (2011) derived (from Equation (7)) a cooling length of $120 \mathrm{au}$ in the inner heliosheath for plasma sampled with ENAs of $1 \mathrm{keV}$ energy, which is appropriate for the upwind heliosheath. For the downwind hemisphere, the energy of $150 \mathrm{eV}$, relevant for the total pressure (Figure 9), implies $v_{\mathrm{ENA}}=\sqrt{2 E / m} \approx 170 \mathrm{~km} \mathrm{~s}^{-1}$ and thus $l_{\text {cool }}=$ $210 \mathrm{au}$. For the lowest-energy bin of $15 \mathrm{eV}$, the cooling length would increase to $430 \mathrm{au}$.

A similar mean free path is found for any heliosheath proton moving through the surrounding neutral hydrogen. The pick-up proton density and the solar wind density are both on the order of only $10^{-3} \mathrm{~cm}^{-3}$ in the inner heliosheath (Richardson et al. 2008; Gloeckler \& Fisk 2011; Reisenfeld et al. 2012). These ions move through the neutral hydrogen with $n_{\mathrm{H}}=0.1$ $\mathrm{cm}^{-3}$, whose speed is only $25 \mathrm{~km} \mathrm{~s}^{-1}$ relative to the Sun (McComas et al. 2015). Therefore,

$$
l_{\text {neutr }}=\frac{1}{n_{\mathrm{H}} \sigma} \approx 220 \mathrm{au} .
$$

The cooling and neutralization lengths of typically $200 \mathrm{au}$ agree for the North, South, and Port Lobe regions with the dimension derived from $P \times l$ within the respective error bars (this study) and from time variations of ENAs (Reisenfeld et al. 2016). Both methods rely on ENA measurements and thus can only sample the cooling length of the plasma rather than 
the full heliosheath thickness. However, Reisenfeld et al. (2016) also noted that these cooling lengths derived from ENA observations are similar to model predictions for the full heliosheath thickness, equal to 100 au (Pogorelov et al. 2013) up to 240 au (Izmodenov et al. 2009) for the north pole region. This implies that, in these directions, IBEX may sample plasma all the way from the termination shock to the heliopause. The pressure method can be used to derive the total heliosheath thickness there if the contributions from low energies around $100 \mathrm{eV}$ are taken into account. Otherwise, the total pressure is underestimated, as shown by Schwadron et al. (2011) $\left(P \times l=72\right.$ pdyn $\mathrm{cm}^{-2}$ au for the $I B E X$-Hi energy range).

The ENA measurements from the Central Tail region suggest that the local neutral hydrogen density is lower than the hitherto assumed $0.1 \mathrm{~cm}^{-3}$ : the emission thickness derived from $P \times l$ exceeds the cooling and neutralization lengths by a factor of $1.7 \pm 0.4$. The excess cannot be remedied by major contributions from energies below $80 \mathrm{eV}$ to the total pressure. This would imply typical energies of 15 or $50 \mathrm{eV}$ and thus stretch the cooling length to roughly $400 \mathrm{au}$. However, this assumption would also increase $P \times l$ (Equation (6)). A region of depleted neutral hydrogen density $n_{H}<0.1 \mathrm{~cm}^{-3}$ extending into the heliosheath around the downwind direction offers the simplest solution to the discrepancy (refer to Equations (7) and (8)). The existence of such a depletion is predicted by stateof-the-art global models of the heliosphere (e.g., Izmodenov et al. 2009; Heerikhuisen et al. 2014). The magnitude of the depletion depends on the strength of the interstellar magnetic field (Heerikhuisen et al. 2014).

The ENA spectra presented here suggest that the neutral hydrogen density in the Central Tail direction cannot exceed $0.1 \times 210 \mathrm{au} / 280 \mathrm{au}$, and more likely drops to $0.06 \mathrm{~cm}^{-3}$ if

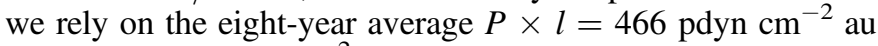
and $P=1.3$ pdyn $\mathrm{cm}^{-2}$. The lower limit could be as low as $0.02 \mathrm{~cm}^{-3}$ if we apply the upper limit of roughly 1000 au from Table 6 . This range of possible neutral densities agrees well with the $\sim 0.06 \mathrm{~cm}^{-3}$ predicted by the simulation of Heerikhuisen et al. (2014) for the central heliotail at an interstellar magnetic field strength of $3 \mu \mathrm{G}$. This field strength is consistent with the $2.93 \pm 0.08 \mu \mathrm{G}$ determined by Zirnstein et al. (2016b) based on the geometry of the ENA Ribbon.

Schwadron et al. (2011) argued that, because the ENA emission thickness cannot exceed the cooling length, the dimension implied by ENA observations was just the cooling length, and $d_{\mathrm{TS}}$ in turn had to be smaller than $145 \mathrm{au}$. With our new analysis, however, this argument no longer limits the termination shock distance to $d_{\mathrm{TS}}<200 \mathrm{au}$.

Our analysis yields a total plasma pressure outside the termination shock of 1.7 pdyn $\mathrm{cm}^{-2}$ with a lower limit of 1.0 pdyn $\mathrm{cm}^{-2}$ toward the heliotail. Inside the termination shock, the total pressure of the solar wind is dominated by the ram term, with the internal pressure of pickup ion and magnetic pressure being the next two smaller contributions at heliocentric distances beyond 50 au (Whang 1998). Let us compare just the ram pressure term with the lower limit of the heliosheath plasma pressure. Because the solar wind density, and thus the ram pressure, drops with the square of the heliocentric distance, the termination shock must be rather close to the Sun also for the downwind hemisphere. We obtain the required minimum pressure of

$$
P=\frac{n_{p} m_{H}}{2} v_{\mathrm{sw}}^{2}=0.1 \mathrm{pP}=1.0 \text { pdyn } \mathrm{cm}^{-2}
$$

with a total proton density of $n_{p}=10^{-3} \mathrm{~cm}^{-3}$ and a bulk flow speed of $340 \mathrm{~km} \mathrm{~s}^{-1}$, which are typical values at $100 \mathrm{au}$ distance (Whang 1998). This is consistent with the $115 \mathrm{au}$ derived for 1.0 pdyn $\mathrm{cm}^{-2}$ by Schwadron et al. (2011) who also included internal pressure. These distances are similar to $d_{\mathrm{TS}}=130 \mathrm{au}$ at the north pole and $d_{\mathrm{TS}}=110 \mathrm{au}$ at the south pole (Reisenfeld et al. 2016). In summary, IBEX observations argue for a rather spherical shape of the termination shock (similar to the model of Izmodenov \& Alexashov 2015, for example).

\section{Conclusions}

We have aggregated all observations of heliospheric ENAs from the downwind hemisphere at low energies measured during the first eight years of the IBEX mission.

1. We confirmed previous studies on heliospheric ENAs: the spectral index of ENA intensity depends on heliolatitude at solar wind energy, but this heliolatitudinal ordering disappears below $0.9 \mathrm{keV}$. The ENA energy spectrum probably has a knee around $0.8 \mathrm{keV}$ and rolls over around $0.1 \mathrm{keV}$ in all downwind directions.

2. We have seen the first indication of temporal changes for low-energy ENAs (0.1 and $0.2 \mathrm{keV})$. The apparent anticorrelation with solar cycle must be revisited once a full solar cycle has been covered. At solar wind energy (0.4-2.5 keV), IBEX-Lo data are insufficient to verify the $30 \%$ changes with time observed with $I B E X$-Hi.

3. The ISN hydrogen signal recovered already in 2014, during solar maximum conditions, earlier than expected.

4. Composite energy spectra from $10 \mathrm{eV}$ to $6 \mathrm{keV}$ for the dynamic pressure times ENA emission thickness in the heliosheath have been compiled for the first eight years of $I B E X$-Lo and seven years of IBEX-Hi data. In the downwind hemisphere, the protons giving rise to heliospheric ENAs around $0.1 \mathrm{keV}$ dominate the total plasma pressure. The study of these ENAs is therefore crucial to understand the vast regions of the heliosphere where no in situ observations are available.

5. Our observations at low energies confirm that the heliosheath toward southern latitudes is compressed compared to northern latitudes and to the Port Lobe.

6. The dynamic pressure of the plasma in the heliosheath reaches 1.7 pdyn $\mathrm{cm}^{-2}$, integrated from $10 \mathrm{eV}$ to $6 \mathrm{keV}$ for any direction in the downwind hemisphere; the lower limit is 1.0 pdyn $\mathrm{cm}^{-2}$.

As a consequence, the thickness of the plasma structures responsible for emission of ENAs in the heliosheath reach 150-210 au toward the poles and flanks, which is similar to the cooling length of the plasma. Because these dimensions agree with other observation methods and model predictions for the total heliosheath thickness, it is possible that IBEX samples ENAs from plasma all the way from the termination shock to the heliopause in all directions except the Central Tail. Here, the plasma pressure from ENA spectra implies an ENA emission thickness of at least $280 \mathrm{au}$. This region coincides with the nominal downwind direction around $\lambda_{\text {ecl }}=76^{\circ}$ longitude. The heliosheath therefore is extended around the downwind direction, compared to the flanks, by at least a factor of 1.4. The upper limit of this shape factor is ill-constrained: ENA intensities measured below $0.1 \mathrm{keV}$ from anti-ram 
direction are affected by large uncertainties, and the heliosheath could be thicker than the plasma cooling length in this region. The derived ENA emission thickness along the IBEX line of sight indicates that the neutral hydrogen is depleted toward the heliotail with respect to other heliosheath regions to densities between 0.02 and $0.075 \mathrm{~cm}^{-3}$.

We are eagerly waiting for the next three years of $I B E X$ observations of 2017,2018 , and 2019, as this will allow us to cover the temporal evolution of heliospheric ENAs over one full solar cycle. The present data suggest that the ENA intensities around $0.1 \mathrm{keV}$ from the downwind hemisphere anticorrelate with solar activity. If confirmed, this implies periodic changes in the plasma pressure and/or the heliosheath dimensions in the downwind hemisphere.

We thank all of the outstanding men and women who have made the IBEX mission such a wonderful success. A.G. and P.W. thank the Swiss National Science foundation for financial support. M.B., M.A.K., and J.M.S. were supported by Polish National Science Center grant 2015-18-M-ST9-00036. H.K., E.M., N.S., H.O.F., S.A.F., and D.J.M. were supported by the NASA Explorer program as a part of the IBEX mission.

\section{ORCID iDs}

A. Galli (i) https://orcid.org/0000-0003-2425-3793

P. Wurz (1) https://orcid.org/0000-0002-2603-1169

M. Bzowski (i) https://orcid.org/0000-0003-3957-2359

H. O. Funsten (1D) https://orcid.org/0000-0002-6817-1039

D. J. McComas (iD https://orcid.org/0000-0002-9745-3502

\section{References}

Burlaga, L. F., Ness, N. F., Acuña, M. H., et al. 2008, Natur, 454, 75 Bzowski, M. 2008, A\&A, 488, 1057

Bzowski, M., Kubiak, M. A., Czechowski, A., \& Grygorczuk, J. 2017, ApJ, 845,15

Decker, R. B., Krimigis, S. M., Roelof, E. C., et al. 2005, Sci, 309, 2020

Desai, M. I., Allegrini, F., Dayeh, M. A., et al. 2015, ApJ, 802, 100

Dialynas, K., Krimigis, S. M., Mitchell, D. G., Decker, R. B., \& Roelof, E. C. , 2017, NatAs, 1, 115

Funsten, H. O., Allegrini, F., Bochsler, P., et al. 2009, SSRv, 146, 75

Fuselier, S. A., Allegrini, F., Bzowski, M., et al. 2012, ApJ, 754, 14

Fuselier, S. A., Allegrini, F., Bzowski, M., et al. 2014, ApJ, 784, 89
Fuselier, S. A., Bochsler, P., Chornay, D., et al. 2009, SSRv, 146, 117

Galli, A., Wurz, P., Fuselier, S. A., et al. 2014, ApJ, 796, 9

Galli, A., Wurz, P., Kollmann, P., et al. 2013, ApJ, 775, 24

Galli, A., Wurz, P., Park, J., et al. 2015, ApJ, 220, 30

Galli, A., Wurz, P., Schwadron, N. A., et al. 2016, ApJ, 821, 107

Gloeckler, G., \& Fisk, L. A. 2011, AIP Conf. Proc. 1302, Pickup Ions throughout the Heliosphere and Beyond, ed. J. A. Le Roux et al. (Melville, NY: AIP), 110

Gloeckler, G., \& Fisk, L. A. 2015, ApJL, 806, L27

Gurnett, D. A., Kurth, W. S., Burlaga, L. F., \& Ness, N. F. 2013, Sci, 341,1489

Heerikhuisen, J., Zirnstein, E. J., Funsten, H. O., Pogorelov, N. V., \& Zank, G. P. 2014, ApJ, 784, 73

Izmodenov, V. V., \& Alexashov, D. B. , 2015, ApJS, 220, 32

Izmodenov, V. V., Malama, Y. G., Ruderman, M. S., et al. 2009, SSRv, 146,329

Kubiak, M. A., Bzowski, M., Sokół, J. M., et al. 2014, ApJS, 213, 29

Kubiak, M. A., Swaczyna, P., Bzowski, M., et al. 2016, ApJS, 223, 25

Lindsay, B. G., \& Stebbings, R. F. 2005, JGR, 110, A12213

Livadiotis, G., McComas, D. J., Schwadron, N. A., Funsten, H. O., \& Fuselier, S. A. 2013, ApJ, 762, 134

McComas, D. J., Allegrini, F., Bochsler, P., et al. 2009, SSRv, 146, 11

McComas, D. J., Bzowski, M., Fuselier, S. A., et al. 2015, ApJS, 220, 22

McComas, D. J., Dayeh, M. A., Funsten, H. O., Livadiotis, G., \& Schwadron, N. A. 2013, ApJ, 771, 77

McComas, D. J., Lewis, W. S., \& Schwadron, N. A. 2014, RvGeo, 52, 118

McComas, D. J., Zirnstein, E. J., Bzowski, M., et al. 2017, ApJS, 229, 41

Möbius, E., Bochsler, P., Bzowski, M., et al. 2012, ApJS, 198, 11

Park, J., Kucharek, H., Möbius, E., et al. 2016, ApJ, 833, 130

Pogorelov, N. V., Seuss, S. T., Borovikov, S. N., et al. 2013, ApJ, 772, 2

Reisenfeld, D. B., Allegrini, F., Bzowski, M., et al. 2012, ApJ, 747, 110

Reisenfeld, D. B., Bzowski, M., Funsten, H. O., et al. 2016, ApJ, 833, 277

Richardson, J. D., Kasper, J. C., Wang, C., et al. 2008, Natur, 454, 63

Saul, L., Bzowski, M., Fuselier, S. A., et al. 2013, ApJ, 767, 130

Schwadron, N. A., Allegrini, F., Bzowski, M., et al. 2011, ApJ, 731, 56

Schwadron, N. A., Möbius, E., Fuselier, S. A., et al. 2014, ApJ, 215, 13

SILSO, World Data Center 2008, Sunspot Number and Long-term Solar Observations, Royal Observatory of Belgium 2008-2014, International sunspot Number Monthly Bulletin and Online Catalogue, http://sidc.be/ silso/

Sokół, J. M., Kubiak, M. A., Bzowski, M., Swaczyna, P., et al. 2015a, ApJS, 220, 27

Stone, E. C., Cummings, A. C., McDonald, F. B., et al. 2013, Sci, 341, 150

Whang, Y. C. 1998, JGR, 103, 17419

Whang, Y. C., Lu, J. Y., \& Burlaga, L. F. 1999, JGR, 104, 28255

Wood, B. E., Izmodenov, V. V., Alexashov, D. B., Redfield, S., \& Edelman, E. 2014, ApJ, 780, 108

Zirnstein, E. J., Dayeh, M. A., McComas, D. J., \& Sokół, J. M. 2017, ApJ, 846, 63

Zirnstein, E. J., Funsten, H. O., Heerikhuisen, J., et al. 2016a, ApJ, 826, 58

Zirnstein, E. J., Heerikhuisen, J., Funsten, H. O., et al. 2016b, ApJL, 818, L18 